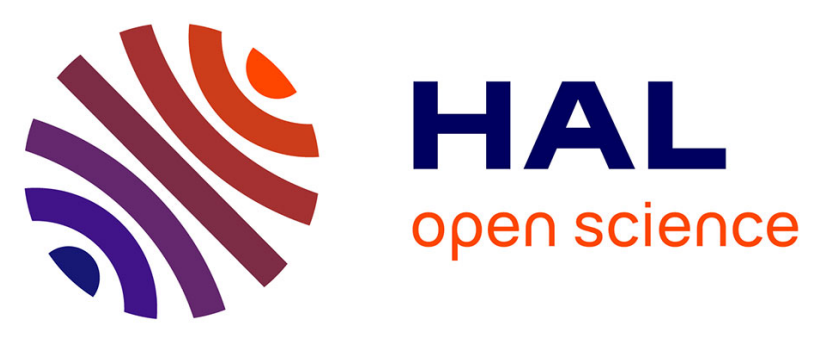

\title{
Detrimental Effect and Neutralization of in Situ Produced Water on Zirconia Nanoparticles Obtained by a Nonaqueous Sol-Gel Method
}

Jess Gambe, Fabien Remondiere, Jenny Jouin, Laura Portal, Philippe Thomas, Olivier Masson

\section{To cite this version:}

Jess Gambe, Fabien Remondiere, Jenny Jouin, Laura Portal, Philippe Thomas, et al.. Detrimental Effect and Neutralization of in Situ Produced Water on Zirconia Nanoparticles Obtained by a Nonaqueous Sol-Gel Method. Inorganic Chemistry, 2019, 58 (22), pp.15175-15188. 10.1021/acs.inorgchem.9b02076 . hal-02433804

\section{HAL Id: hal-02433804 \\ https://hal-unilim.archives-ouvertes.fr/hal-02433804}

Submitted on 26 Nov 2020

HAL is a multi-disciplinary open access archive for the deposit and dissemination of scientific research documents, whether they are published or not. The documents may come from teaching and research institutions in France or abroad, or from public or private research centers.
L'archive ouverte pluridisciplinaire HAL, est destinée au dépôt et à la diffusion de documents scientifiques de niveau recherche, publiés ou non, émanant des établissements d'enseignement et de recherche français ou étrangers, des laboratoires publics ou privés. 
This document is confidential and is proprietary to the American Chemical Society and its authors. Do not copy or disclose without written permission. If you have received this item in error, notify the sender and delete all copies.

\section{Detrimental effect and neutralization of in situ produced water on zirconia nanoparticles obtained by a nonaqueous sol-gel method}

\begin{tabular}{|r|l|}
\hline Journal: & Inorganic Chemistry \\
\hline Manuscript ID & ic-2019-02076t.R2 \\
\hline Danuscript Type: & Article \\
\hline Complete List of Authors: & $\begin{array}{l}\text { Gambe, Jess; Institut de Recherche sur les Céramiques } \\
\text { REMONDIERE, Fabien; Institut de Recherche sur les Céramiques } \\
\text { Jouin, Jenny; Institut de Recherche sur les Céramiques } \\
\text { Portal, Laura; Institut de Recherche sur les Céramiques } \\
\text { Thomas, Philippe; Institut de Recherche sur les Céramiques } \\
\text { Masson, Olivier; Institut de Recherche sur les Céramiques }\end{array}$ \\
\hline \multicolumn{2}{|}{} \\
\hline
\end{tabular}

\section{SCHOLARONE Manuscripts}




\title{
Detrimental effect and neutralization of
}

\section{in-situ-produced water on zirconia nanoparticles}

\author{
obtained by a nonaqueous sol-gel method
}

\author{
Jess Gambe, Fabien Rémondière *, Jenny Jouin, Laura Portal, Philippe Thomas and Olivier \\ Masson \\ Institute of Research for Ceramics (IRCER), UMR 7315 CNRS, Université de Limoges, Centre \\ Européen de la Céramique, 12 rue Atlantis, 87068 Limoges Cedex, France
}

KEYWORDS: Zirconia nanoparticles, nonaqueous sol-gel route, alcohol dehydration, in-situ water production.

\section{Supporting Information}

\begin{abstract}
In this work, the phase purity and size of zirconia nanocrystals samples were studied in terms of zirconium concentration, added water content and subsequent use of a post solvothermal treatment. The progressive tetragonal-to-monoclinic transformation of zirconia sample was observed to be strongly related to the water content of the alcoholic medium. But more surprisingly, it has been shown that even under initially anhydrous conditions and for particle size below $5 \mathrm{~nm}$, the phase purity of the samples was deteriorated by a side-reaction of alcohol dehydration catalyzed by the surface of the nanoparticles (NPs). Since the phase transformation is essentially driven by the water content of the reaction mixture, we have shown that it was possible to recover an excellent phase purity without the help of any usual dopants
\end{abstract}


by adding a strong alkaline desiccating agent. Provided that a sufficient sodium to zirconium ratio was ensured, the formation of the monoclinic phase was not observed whatever the zirconium precursor concentration. The effectiveness of this cure was related to the ability of sodium metal to generate reactive alkoxide able to neutralize water and to catalyze an alternative sol-gel mechanism leading to the formation of the $\mathrm{t}-\mathrm{ZrO}_{2} \mathrm{NPs}$.

\section{INTRODUCTION}

Zirconia $\mathrm{ZrO}_{2}$ is a ceramic oxide with three usual polymorphs at standard pressure, i.e., monoclinic $(\mathrm{m})$, tetragonal $(\mathrm{t})$, and cubic $(\mathrm{c})$. In the bulk, zirconia is stable as the monoclinic phase at room temperature and then, as the temperature increases, it transforms into the tetragonal phase $\left(1170^{\circ} \mathrm{C}<\mathrm{T}<2370^{\circ} \mathrm{C}\right)$ and, eventually, into the cubic phase above $2370^{\circ} \mathrm{C}$. ${ }^{1}$ The cubic phase has the fluorite structure, and the other polymorphs are distorted versions of this structure. Zirconia finds application in many technologies, e.g. solid-state gas sensors, electrolytes for solid oxide fuel cells, thermal barriers, structural material, catalysis... ${ }^{2-5}$ In most of these applications, zirconia must be stabilized at room temperature in the tetragonal or cubic form. This can be achieved by two methodologies: (i) by incorporating cation dopants - the inclusion of a divalent, trivalent or tetravalent cation of a size different from that of $\mathrm{Zr}^{4+}$ (e.g. $\mathrm{Mg}^{2+}, \mathrm{Ca}^{2+}, \mathrm{Y}^{3+}, \mathrm{Ce}^{4+}$ ) induces the apparition of oxygen vacancies that stabilizes the high symmetry phases; and (ii) by limiting the crystallite size below the critical size which induces the stabilization of the metastable polymorph at room temperature due to differences in surface energies. ${ }^{1,6-9}$ Garvie predicted that pure $\mathrm{ZrO}_{2}$ could be stabilized in the tetragonal structure at room temperature when the spherical particle size is less than $30 \mathrm{~nm}$. Such effect has been experimentally shown by many authors. ${ }^{10-14}$ As a catalyst, $\mathrm{ZrO}_{2}$ has been selected due to its specific amphoteric behavior and large thermal stability in order to catalyze reactions including 
the dehydration and dehydrogenation of alcohols, hydrogenation and isomerization of olefins. Among the usual polymorphs, the tetragonal polymorph $\mathrm{t}-\mathrm{ZrO}_{2}$ is believed to possess the highest catalytic activity. ${ }^{15-21}$

For the preparation of inorganic materials with well-defined morphologies, soft chemistry syntheses are preferred to the solid-state chemistry methodology. Over the past two decades, several chemical methods have been developed for the preparation of zirconia nanocrystals, including sol-gel, thermal decomposition, aqueous precipitation with or without hydrothermal treatment, and recently a two-phase approach based on the phase transfer and a separation mechanism. ${ }^{22}$ Another possible general method is the nonhydrolytic sol-gel route successfully employed in the presence of surfactant by Joo et al. or via a glycothermal treatment by Inoue et al. or via a surfactant-free route by Garnweitner et al.. ${ }^{23-27}$ There are many ways to prepare $\mathrm{ZrO}_{2}$ particles, but solvothermal methods have attracted more attention in recent years, especially the benzyl alcohol route, which is a surfactant-free but solvent-controlled route enabling the production of high purity compounds with good control over the particle size, the surface properties, and the assembly behavior. ${ }^{28}$

During the solvothermal synthesis of nanoparticles, even if the precursors, the additives and the solvent are water-free, it is not impossible that some water is produced by secondary reactions and mainly contributes to the formation mechanism of the final oxide nanoparticles; indeed, the presence of water has been detected in various chemical systems and authors prefer thus to refer to the denomination of nonaqueous sol-gel route. ${ }^{29-34}$ Moreover, the producedwater is known to be able to activate the condensation of metallic precursors or clusters and to initiate the crystallization of metal oxide nanoparticles. Only a few articles are focused on the potential effect of water during synthesis and studied its influence on the precursor condensation, the size of the final nanoparticles and more importantly, the polymorphic variety that results. ${ }^{35}$ 
In this paper, we present the effect of in-situ formation of water, which degrades the phase purity and the size control of zirconia nanoparticles obtained by the nonaqueous benzyl alcohol route developed by Garnweitner. ${ }^{26}$ In a first part, we investigate the effect of the zirconium precursor concentration, the water content intentionally introduced into the reaction mixture and finally, the subsequent addition of a post-solvothermal treatment onto as-synthesized NPs. In a second part, we study the use of a reactive sodium alkoxide as an effective cure against the detrimental effect of water on the sample phase purity. The possible roles of sodium cations and alkoxide species are finally discussed and taken in account to propose an original NP formation pathway.

\section{EXPERIMENTAL SECTION}

All chemicals were stored and used as received in a dried-air flow glovebox. Water in the solvents was titrated by the Karl Fischer method and all the solvents were found to be strictly anhydrous. For the solvothermal treatment, Berghof digestion bombs were used with $25 \mathrm{~mL}$ inner volume Teflon cup.

Two series of samples have been synthesized and differ essentially by the presence or absence of a sodium precursor. The experimental conditions are summarized in Table 1. The first series of samples was prepared by introducing 1,4 or $32 \mathrm{mmol}$ of zirconium isopropoxide isopropanol adduct $\left[\mathrm{Zr}\left(\mathrm{O}^{\mathrm{i} P r}\right)_{4} \cdot \mathrm{HO}^{\mathrm{i} P r}\right]_{2}($ Aldrich, $99.9 \%)$ and $12.5 \mathrm{~mL}$ of anhydrous benzyl alcohol $\mathrm{BnOH}$ (Aldrich, 99.8\% anhydrous) into a Teflon reactor. For selected cases, the syntheses were repeated in aqueous conditions with the use of a mixed solvent (deionized water and benzyl alcohol) in replacement of pure anhydrous benzyl alcohol. In addition, the effect of a solvothermal post-treatment for 48 hours at $210^{\circ} \mathrm{C}$ in anhydrous or partially benzyl alcohol medium, namely with an aqueous fraction of 5, 20 and 50 vol.\%, was tested on nanoparticles prepared with the initial anhydrous conditions. The second series of samples were produced via 
an alternate procedure consisting in adding the predissolution step of a sodium precursor, namely sodium metal $\mathrm{Na}$ (Alfa Aesar, 99.95\%), sodium isopropoxide $\mathrm{Na}^{\mathrm{i} O P r}$ (Alfa Aesar, 99\%) or sodium chloride $\mathrm{NaCl}$ (Alfa Aesar, puratronic 99.998\%). It is important to note that unlike $\mathrm{Na}^{\mathrm{i}} \mathrm{OPr}$ which is soluble for a concentration of 0.20 mol. $\mathrm{L}^{-1}, \mathrm{NaCl}$ is only poorly soluble into benzyl alcohol and was directly added to the initial zirconium alkoxide benzyl alcohol mixture. The dissolution of sodium metal in benzyl alcohol $(\mathrm{BnOH})$ occurred at room temperature under the slow release of hydrogen gas as it is shown in the following redox reaction:

$$
\mathrm{Na}_{(s)}+\mathrm{BnOH}_{(l)} \rightarrow \mathrm{NaOBn} n_{(\text {solv })}+\frac{1}{2} \mathrm{H}_{2(g)}
$$

$\mathrm{NaCl}$ and $\mathrm{Na}^{\mathrm{i}} \mathrm{OPr}$ were used with a solute concentration of $0.20 \mathrm{~mol}_{\mathrm{L}} \mathrm{L}^{-1}$ whereas the sodium benzoxide solution produced in the case of sodium metal and benzyl alcohol was studied for various concentrations, i.e. $0.05,0.10,0.20,0.40$ or $1.00 \mathrm{~mol} . \mathrm{L}^{-1}$. A volume of $12.5 \mathrm{~mL}$ of the prepared sodium-based solution was then used as solvent in addition to zirconium isopropoxide isopropanol adduct (4 mmol).

Whatever the initial reaction mixture (series 1 or 2), the Teflon reactor was inserted and sealed into the inox steel bomb, and heated up to $210^{\circ} \mathrm{C}$ for 48 hours via a pre-heated isothermal apparatus disposed on a hot plate. After cooling, the milky suspension was centrifuged and the precipitate was washed three times thoroughly with $20 \mathrm{~mL}$ anhydrous ethanol, dispersed in dichloromethane and finally air-dried over the night at room temperature.

The obtained samples were characterized by the following techniques: X-ray powder diffraction (XRD), transmission electron microscopy (TEM), selected area electron diffraction (SAED), thermogravimetric analysis (TG), ${ }^{1} \mathrm{H}$ - and ${ }^{13} \mathrm{C}$-based nuclear magnetic resonance (NMR) in the case of the study of the supernatant diluted in DMSO-d6 and elemental analysis using inductively coupled mass spectroscopy (ICP-MS). The XRD diagrams of all the samples 
were collected using a Brucker D8 Advance diffractometer, operating in the Bragg-Brentano configuration using $\mathrm{Cu} \mathrm{K} \mathrm{K}_{\alpha 1}$ radiation $(\lambda=1.54 \AA)$ from $10^{\circ}$ to $110^{\circ}$. The size, morphology and distribution in size of the samples were analyzed using a JEOL JEM-2100F microscope at an accelerating voltage of $200 \mathrm{kV}$. Specimens for the TEM studies were prepared by depositing a drop of diluted suspensions onto a 400 mesh copper grid, coated with an ultrathin lacey carbon film. Prior to deposition, the suspensions were sonicated for $2 \mathrm{~min}$ to avoid excessive aggregation and diluted into dichloromethane $\left(\mathrm{CH}_{2} \mathrm{Cl}_{2}\right)$ in the presence of oleic acid. The thermogravimetric analyses were performed on a Netzsch instrument (model STA 449F3, Germany) and carried out from $25^{\circ} \mathrm{C}$ to $800^{\circ} \mathrm{C}$ at a heating rate of $10^{\circ} \mathrm{C} \cdot \mathrm{min}^{-1}$ in flowing argon gas (20 mL.min $\left.{ }^{-1}\right)$. ICP-MS was accomplished using a Perkin-Elmer Sciex Elan 6100 instrument to evaluate the relative amount of sodium compared to zirconium. Prior to the elemental analysis, the nanoparticles were first calcined at $900^{\circ} \mathrm{C}$ and then dissolved using a microwave-assisted digester in the presence of concentrated sulfuric, nitric and hydrofluoric acids. The resulted solutions were then diluted in the presence of boric acid to enhance the solutions stability.

Table 1. Experimental conditions used for the synthesis of zirconia nanoparticles prepared at $210^{\circ} \mathrm{C}$ for 48 hours with zirconium isopropoxide-isopropanol adduct in anhydrous benzyl alcohol (or aqueous benzyl alcohol), monoclinic phase content and average nanoparticles size.

\begin{tabular}{|c|c|c|c|c|c|c|c|c|}
\hline \multirow{2}{*}{$\begin{array}{c}{[\mathrm{Zr}]} \\
\left(\mathrm{mol} . \mathrm{L}^{-1}\right)\end{array}$} & \multirow{2}{*}{$\begin{array}{l}\text { Sodium } \\
\text { source }\end{array}$} & \multirow{2}{*}{$\begin{array}{c}{[\mathrm{Na}]} \\
\left(\mathrm{mol} \cdot \mathrm{L}^{-1}\right)\end{array}$} & \multirow[t]{2}{*}[\mathrm{Na}]{$/[\mathrm{Zr}]$} & \multirow{2}{*}{$\begin{array}{l}\text { Added } \\
\text { water } \\
\text { (vol.\%) }\end{array}$} & \multicolumn{2}{|c|}{$\begin{array}{l}\text { Solvothermal } \\
\text { post-treatment }\end{array}$} & \multirow{2}{*}{$\begin{array}{c}\mathrm{m}^{-\mathrm{ZrO}_{2}} \\
\text { content } \\
(\mathrm{wt} . \%)\end{array}$} & \multirow{2}{*}{$\begin{array}{l}\text { NPs } \\
\text { size } \\
(\mathrm{nm})\end{array}$} \\
\hline & & & & & Temp./time & $\begin{array}{c}\text { water } \\
\text { content } \\
\text { (vol.\%) }\end{array}$ & & \\
\hline 0.08 & none & 0 & 0 & 0 & none & none & 37 & 3.3 \\
\hline 0.32 & none & 0 & 0 & 0 & none & none & 44 & 3.7 \\
\hline 1.28 & none & 0 & 0 & 0 & none & none & 74 & 4.5 \\
\hline 0.32 & none & 0 & 0 & 5 & none & none & 51 & 5.2 \\
\hline
\end{tabular}




\section{RESULTS AND DISCUSSION}

\section{III.1. Detrimental effect of water on zirconia nanocrystals}

\section{a) Effect of zirconium isopropoxide concentration on sample phase purity}

Three syntheses were carried out in anhydrous benzyl alcohol with different amounts of zirconium isopropoxide isopropanol adduct of respectively $0.08,0.32$ and $1.28 \mathrm{~mol}^{-\mathrm{L}^{-1}}$. The XRD diagrams of the samples are shown in the Figure 1. The three samples are clearly crystallized and the peak broadening indicates the nanocrystalline nature of the particles. For the sample obtained with the lowest concentration (Figure 1(a)), the main peaks can be attributed to the tetragonal phase, denoted here $\mathrm{t}-\mathrm{ZrO}_{2}$, (space group P4 $4_{2} / \mathrm{nmc}$ - JCPDS 00-0501089). However, since the tetragonal phase of zirconia is slightly distorted with respect to the 
fluorite cubic phase (for bulk $\mathrm{ZrO}_{2}$, the c/a ratio is only 1.023 , very close to the cubic ideal value of 1.0) it is very difficult to distinguish between these the tetragonal phase and a pseudocubic one. The extra peaks at $2 \theta=24.0^{\circ}-24.5^{\circ}, 40.7^{\circ}-41.3^{\circ}$ and $44.8^{\circ}-45.5^{\circ}$ unambiguously evidences the presence of the monoclinic polymorph $\mathrm{m}-\mathrm{ZrO}_{2}\left(\right.$ Baddeleyite $\mathrm{P} 2{ }_{1} / \mathrm{a}-\mathrm{JCPDS} 00$ 037-1484) which cannot explain by itself all the peaks of the diagram. Moreover, the peaks intensity of $\mathrm{m}-\mathrm{ZrO}_{2}$ is becoming more pronounced as the concentration of zirconium isopropoxide increases from 0.08 up to $1.28 \mathrm{~mol}^{-L^{-1}}$ (Figure $1(\mathrm{a}-\mathrm{c})$ ).

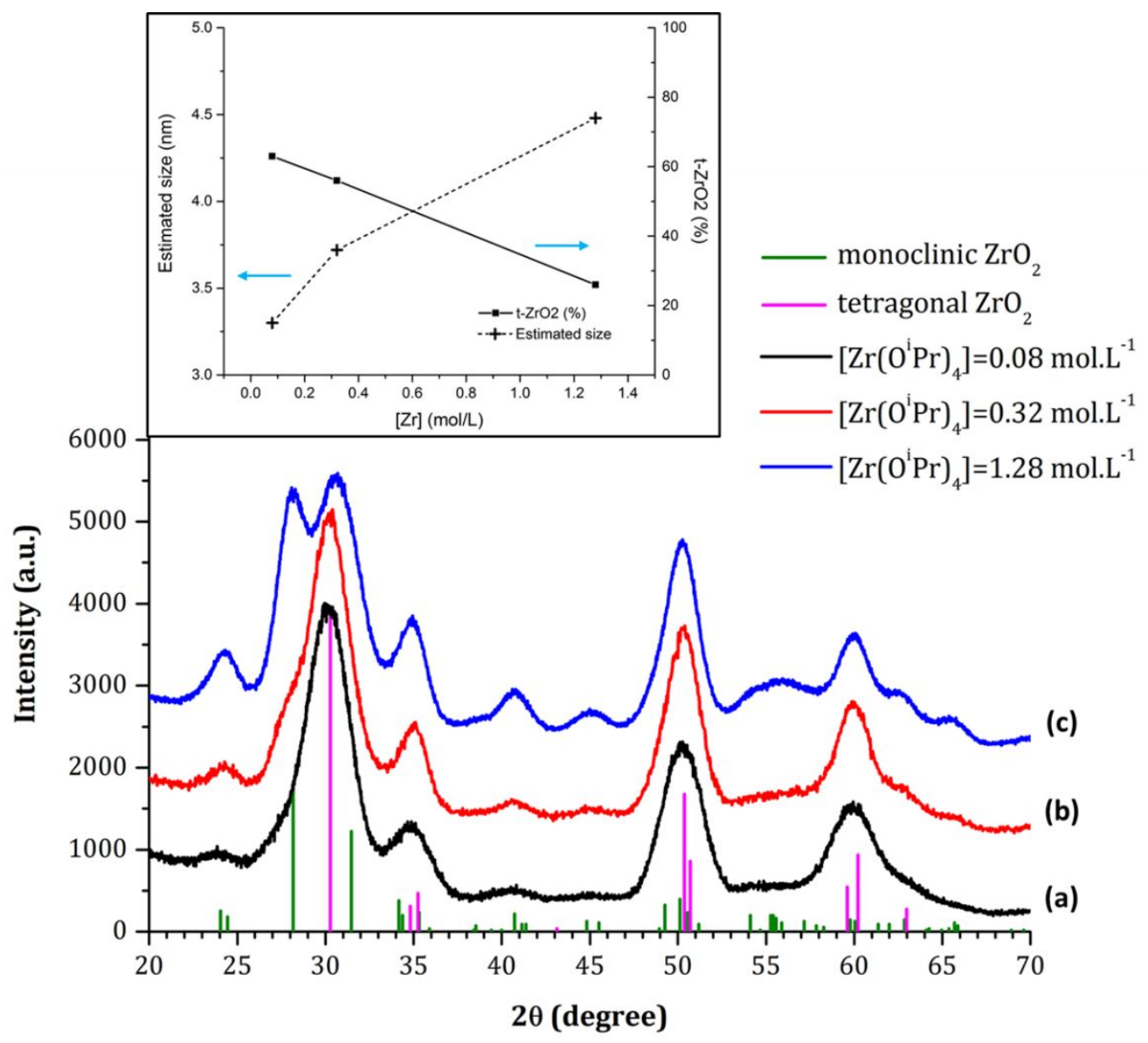

Figure.1. X-ray diffraction patterns of $\mathrm{ZrO}_{2}$ nanoparticles prepared by the Na-free benzyl

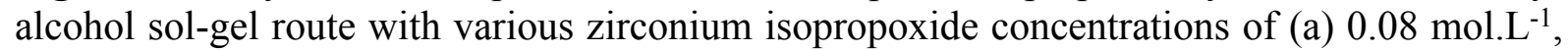
(b) $0.32 \mathrm{~mol} . \mathrm{L}^{-1}$ and (c) $1.28 \mathrm{~mol} . \mathrm{L}^{-1}$. Also shown are the estimated size and $\mathrm{t}-\mathrm{ZrO}_{2}$ weight fraction (inset).

In order to determine the average crystallite size and to evaluate the relative proportion of each phases, a Rietveld refinement was performed for the three samples. Due to the large peaks 
broadening and overlap the refined peak profile and width parameters were constrained to be equal during the calculation assuming the same particle size for both $\mathrm{t}-$ and $\mathrm{m}-\mathrm{ZrO}_{2}$ phases. This assumption on the crystallite size will be accredited by the TEM study of the sample. The addition of microstrains is not needed to perform the Rietveld refinement. An example of refinement is given in the Figure 2(a) and the global results are gathered in the Table 1. The average crystallite size was found to be $3.3 \mathrm{~nm}\left(37 \mathrm{wt} . \%\right.$ of m- $\left.\mathrm{ZrO}_{2}\right), 3.7 \mathrm{~nm}(44 \mathrm{wt} \%$ of m$\left.\mathrm{ZrO}_{2}\right)$ and $4.5 \mathrm{~nm}\left(74 \mathrm{wt} . \%\right.$ of $\left.\mathrm{m}-\mathrm{ZrO}_{2}\right)$ for the concentration of $0.08 \mathrm{~mol} . \mathrm{L}^{-1}, 0.32 \mathrm{mol.L}-1$ and finally $1.28 \mathrm{~mol} . \mathrm{L}^{-1}$ respectively. These results indicate a significant and strong dependence of the precursor concentration on the resulting nanoparticle size and the relative fraction of each polymorphs. Even the use of a quite low concentration in zirconium isopropoxide is not sufficient to guaranty a good phase purity of the resulting nanoparticles. TEM investigation was undertaken on the sample prepared with a nominal zirconium isopropoxide concentration of $0.32 \mathrm{~mol} . \mathrm{L}^{-1}$; the Figure 2(b) corresponds to the analysis of the wet particles dispersed onto an ultra-thin carbon membrane. The overview micrograph of the grid at intermediate magnification illustrates that the wet particles entirely consist of monodisperse objects measuring a few nanometers. The inset image exhibits a crystallized nanoparticle measuring $3.8 \mathrm{~nm}$. The average particle diameter measured on the large overview is estimated to $3.5+/-0.5 \mathrm{~nm}$ and close to the Rietveld calculation. 

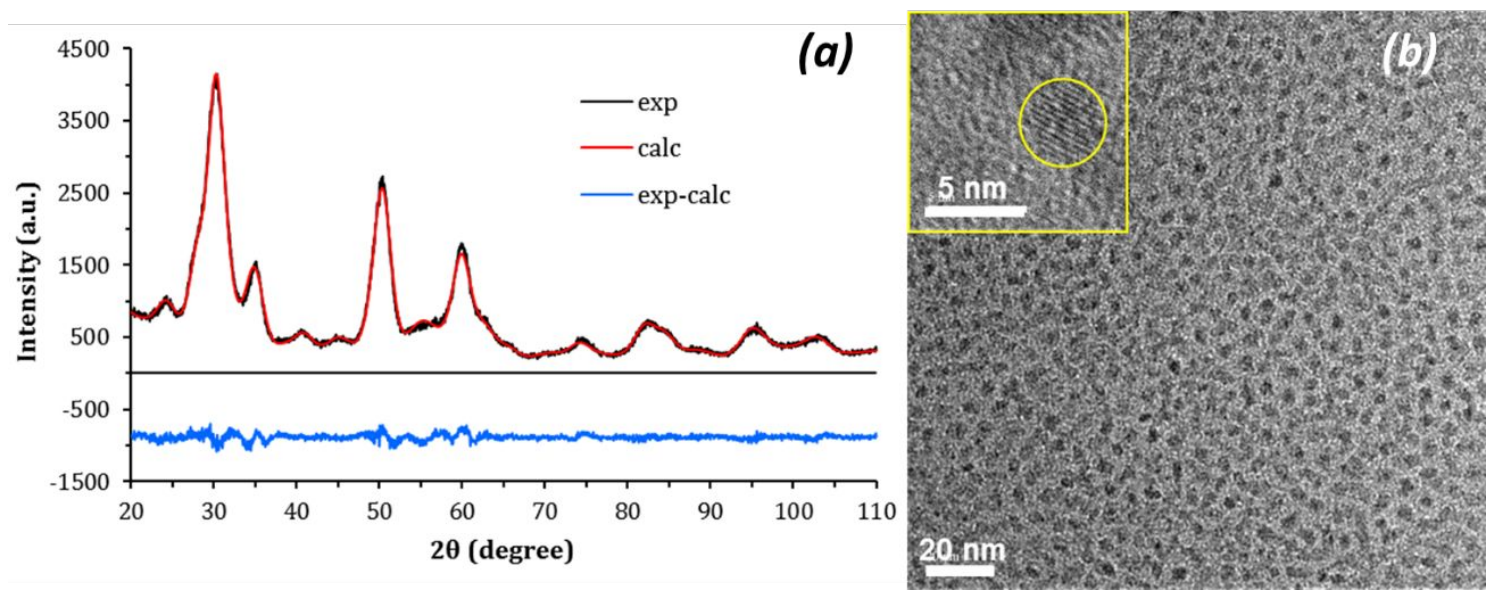

Figure.2. Rietveld refinement of the XRD pattern of the sample prepared by the Na-free benzyl alcohol sol-gel route with a nominal concentration in zirconium isopropoxide of $0.32 \mathrm{~mol} . \mathrm{L}^{-1}$ (a) and TEM micrograph of the wet nanoparticles dispersed onto a ultra-thin carbon membrane (inset: HRTEM view of a single crystallized nanoparticle) (b).

Niederberger and coworkers have shown that the investigation of nanoparticle formation has to be considered from both the inorganic and the organic sides. In that perspective, the supernatant liquid corresponding to the reference 0.32 mol.L-1 sample was collected after centrifugation and subjected to ${ }^{13} \mathrm{C}$ - and ${ }^{1} \mathrm{H}-\mathrm{NMR}$ analyses revealing the major presence of benzyl alcohol and isopropanol, and in smaller amounts the presence of benzyl and diisopropyl ethers and water (Figure.S1. in supporting information). The presence of ethers is consistent with the results of Garnweitner et al. indicating that the main source for zirconia formation is an ether-elimination condensation mechanism. ${ }^{26}$ Besides the residual alcohols and the resulting ethers, the presence of water is quite surprising for a nonaqueous solvothermal route based on an aprotic condensation reaction and was confirmed by a positive copper (II) sulfate test. Since the main condensation mechanism proposed by Garnweitner et al. is a two-step process involving ligand exchange reaction in the presence of large excess of benzyl alcohol and second, aprotic ether-elimination, no formal water can be released and no hydroxyl group appears during the formation of the oxidic network. Among the other possible condensation reactions that have been observed and referenced by Niederberger, just a few of them occur via 
the formation of hydroxylated species and thus the possible release of water, i.e. either the thermal decomposition of isopropoxide ligands, metal halide reaction with alcohols, aldol condensation or the C-C coupling of isopropoxide ligand with benzyl alcohol analogously to what occurs in Guerbet reaction. In the absence of zirconium halide or ketones, we can restrain the perimeter to thermal decomposition and C-C coupling reactions. ${ }^{34}$ Since the thermal decomposition of metal alkoxide necessitates the use of a temperature as high as $350^{\circ} \mathrm{C}$, we can exclude such eventuality in our case. The C-C coupling of benzyl alcohol and isopropoxide ligand resulting in the formation of hydroxylated species cannot be evocated to supply the formal water because on the one hand zirconium alkoxides are not known to exhibit a high Lewis acidity contrary to yttrium cation and on the other hand no coupling products of isopropyl and benzyl alcohol (e.g. 4-phenyl-2-butanol) were found in the supernatant. ${ }^{36-38}$

If water is not produced by the condensation reaction leading to the formation of the zirconia nanoparticles, it is necessary to take in account some plausible parasitic reactions with the solvent. Commonly the alcohols dehydrate in the presence of a strong acid to form either ethers at low temperatures, e.g. Williamson ether synthesis, or alkenes at higher temperatures. Inspired by the work of Kotestkyy, we propose that the water could also originate from the dehydration of the alcoholic medium mediated by the catalytic properties of the growing surface of the zirconia nuclei or surface of zirconia nanocrystals. ${ }^{39}$ Kotestkyy et al. proposed the following sequence in the case of the Lewis-acid catalyzed dehydration of simple alcohols on various simple metal oxide like $\mathrm{TiO}_{2}, \mathrm{ZrO}_{2}$ and $\gamma-\mathrm{Al}_{2} \mathrm{O}_{3}$ : (i) alcohol adsorption on the Lewis acid metal site, (ii) $\beta$-hydrogen transfer to a surface $O$, (iii) alkene formation and desorption, (iv) water formation, desorption and catalyst regeneration. In the present case, intermolecular dehydration for benzyl alcohol and inter- and/or intramolecular dehydration for isopropanol could be evocated to argue the release of water during the solvothermal treatment. ${ }^{40-41}$ Since ethers were found in minor proportion in the supernatant we can exclude the intermolecular dehydration of 
alcohols and focus our attention on the intramolecular dehydration of isopropanol supported by the nanoparticle surface. Isopropanol provided by the isopropoxide-isopropanol adduct or issued from the ligand exchange reaction with benzyl alcohol seems to interact with an acidbase couple site of the oxidic catalyst, namely the zirconia nanoparticles, to dehydrate into water and volatile propene which eliminates at the opening of the reactor.

\section{b) Detrimental effect of water on sample purity}

In order to investigate whether the presence of in situ water has a significant impact on the stabilization or the formation of the monoclinic polymorph of zirconia in the final product, we decided to perform two series of experiments: (i) an intentional addition of water into the initial reaction mixture, (ii) a subsequent solvothermal treatment of the as-synthesized wet nanoparticles in regenerated anhydrous or partially hydrated benzyl alcohol.

The first series of syntheses were performed with the preliminary addition of 5, 10 and 20 vol.\% of deionized water in benzyl alcohol. The XRD diagrams of the resulting samples are gathered in the Figure 3. By increasing the content of water in the initial reaction mixture, we observe a modification in the peak broadening and the clear emergence of the monoclinic signature. Rietveld refinements were done to evaluate the average crystallite size and the phase composition of the samples. A significant increase in the nanoparticle size from $3.7 \mathrm{~nm}$ up to $7.0 \mathrm{~nm}$ is observed when $20 \mathrm{vol} \%$ is added in benzyl alcohol but the nanometric nature of the samples appears to be maintained despite the presence of water in the initial reaction mixture. With regards to the respective fraction of each polymorph within the samples, the action of the added water into the initial reaction mixture and in particular the influence of its content seem to be a more modest factor than that of the concentration of alkoxide seen previously in the section III.1.a). Indeed, the phase distribution is not greatly disturbed and the proportion of m$\mathrm{ZrO}_{2}$ nanocrystals increases from $44 \%$ to $51 \%$ when 5 vol. $\%$ water is added, then reaches $59 \%$ 
with a water addition of 10 vol. \%, and no longer evolve in the case of a water addition of 20 vol.\%. The applied parameters do not permit a full production of $\mathrm{m}-\mathrm{ZrO}_{2}$ nanocrystals. In the work of De Keukeleere et al., in which the syntheses were performed with an extremely short reaction time and a low concentration of $0.13 \mathrm{mol.L} \mathrm{L}^{-1}$ for zirconium isopropoxide, no drastic change into the crystal phase was noticed for a water volume ratio of 11 vol.\%. ${ }^{35}$ Nevertheless, we can distinguish in their diagram the raise of a minor impurity corresponding to $\mathrm{m}-\mathrm{ZrO}_{2}$, especially at $24-24.5^{\circ}$ and at $28^{\circ}$ at the bottom of the main broad peak of c- $\mathrm{ZrO}_{2}$. We have to note that the use of a low concentrated mixture and a short reaction time which reduces de facto the in-situ ageing time of the nanoparticles in the presence of water could be the reason why the crystal change is not enough noticeable in their case.

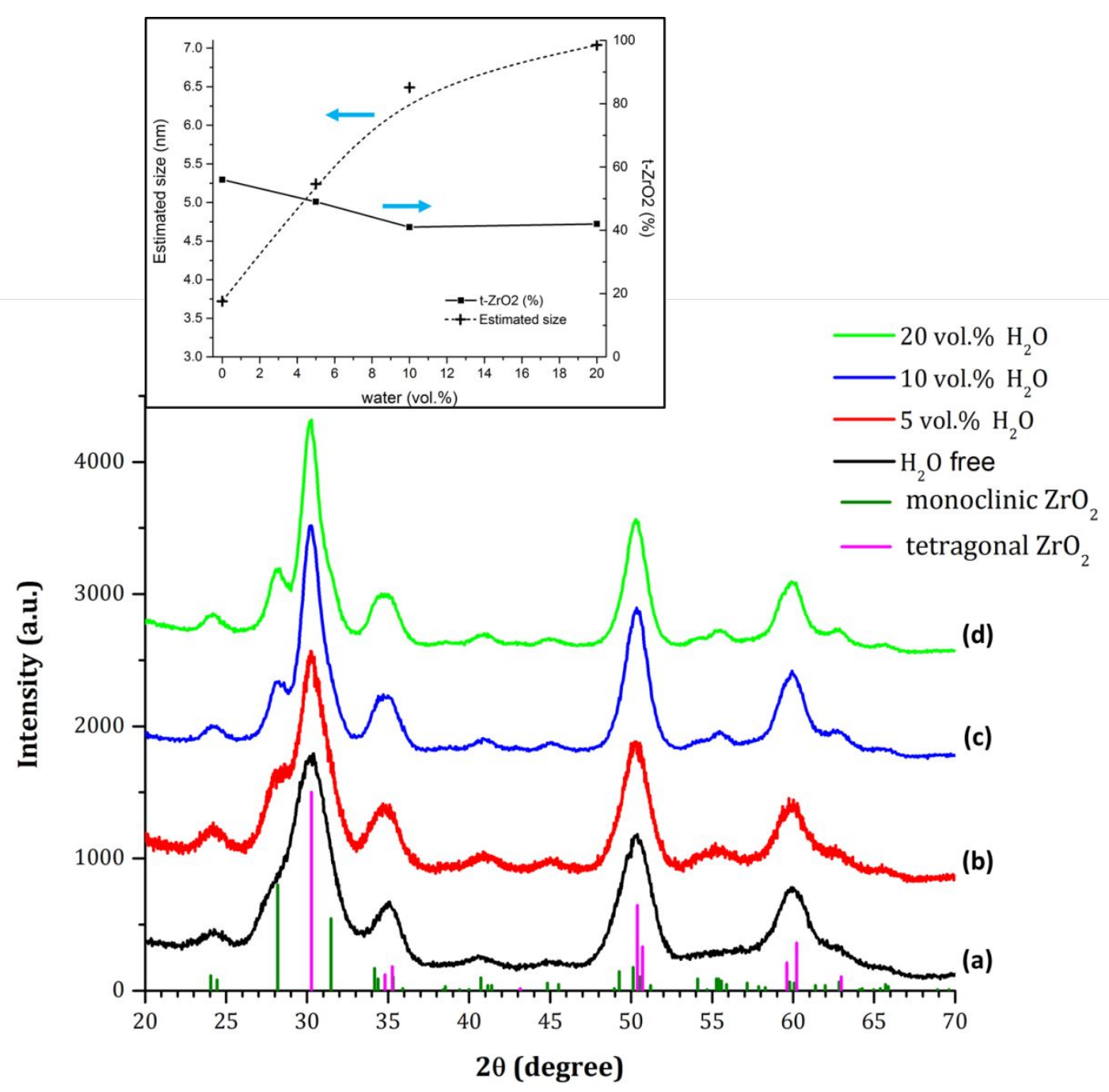


Figure.3. X-ray diffraction patterns of $\mathrm{ZrO}_{2}$ nanoparticles prepared by the Na-free benzyl alcohol sol-gel route in the presence of deionized water, i.e. anhydrous (a), 5 vol.\% (b), 10 vol.\% (c) and 20 vol.\% (d) . Also shown are the estimated size and $\mathrm{t}-\mathrm{ZrO}_{2}$ weight fraction (inset).

The initial presence of water into the reaction mixture essentially induces a growth of the nanoparticles and affects to a lesser extent the polymorphic distribution of the sample showing that the hydrolytic sol-gel reactions are activated and able to absorb a certain hydration of the medium. It is not possible to conclude precisely on the effect of the presence of water during the synthesis and especially the formation of the nanocrystals because the metal precursor modification toward inorganic clusters, the nucleation of the oxidic material, the particle growth and finally the ageing of the particles are not distinguishable but overlapped steps. We can just underline that the presence of water by means of its ionizing, dispersing and solvating actions will drastically modify the surface chemistry of the particles.

To assess the effect of a forced ageing in anhydrous or partially aqueous medium, assynthesized wet particles were submitted to a subsequent solvothermal post-treatment for at least 48 hours at $210^{\circ} \mathrm{C}$. The reaction medium of the second treatment consisted of either anhydrous benzyl alcohol or a mixture of benzyl alcohol and deionized water in volume ratios of 5, 20 and 50 vol.\%. The resulting aged-powders were analyzed by XRD and the results are summarized in the Figure 4. The witness experiment obtained after a second treatment in anhydrous benzyl alcohol certifies that the anhydrous solvothermal post-treatment has only minor effect on the phase purity since the proportion of $\mathrm{m}-\mathrm{ZrO}_{2}$ nanocrystals is around $41 \mathrm{wt} . \%$ and almost the same than at the end of the initial solvothermal treatment. The particle size of the crystalline sample is evaluated at $3.7 \mathrm{~nm}$ and just reflects a small crystallite growth. Once water is added for the solvothermal post-treatment, the XRD diagrams show that the presence of water into the mixture plays a key role in the monoclinic content of the aged-particles. Indeed 5 vol.\% of water as co-solvent appears to be sufficient to modify the nature of the sample and 
thus to change the shape of the diffraction pattern including the shoulder evidenced at $28^{\circ}$. The proportion of $\mathrm{m}-\mathrm{ZrO}_{2}$ nanocrystals reaches almost $57 \mathrm{wt} . \%$. When the water content reaches a value of 20 vol.\% the sample appears to be largely consisting of crystalline nanoparticles of monoclinic variety; indeed, the proportion of $\mathrm{m}-\mathrm{ZrO}_{2}$ nanocrystals is around 84 wt.\%. Posttreatment in the presence of a large quantity of water (50 vol.\%) does not change the particle size of the monoclinic phase neither the purity of the phase since the proportion of $\mathrm{m}-\mathrm{ZrO}_{2}$ nanocrystals is around 89 wt.\% probably due to the fact that water and benzyl alcohol are only partially miscible even at $210^{\circ} \mathrm{C}$ and this limits the hydrolysis of the organophilic particles.

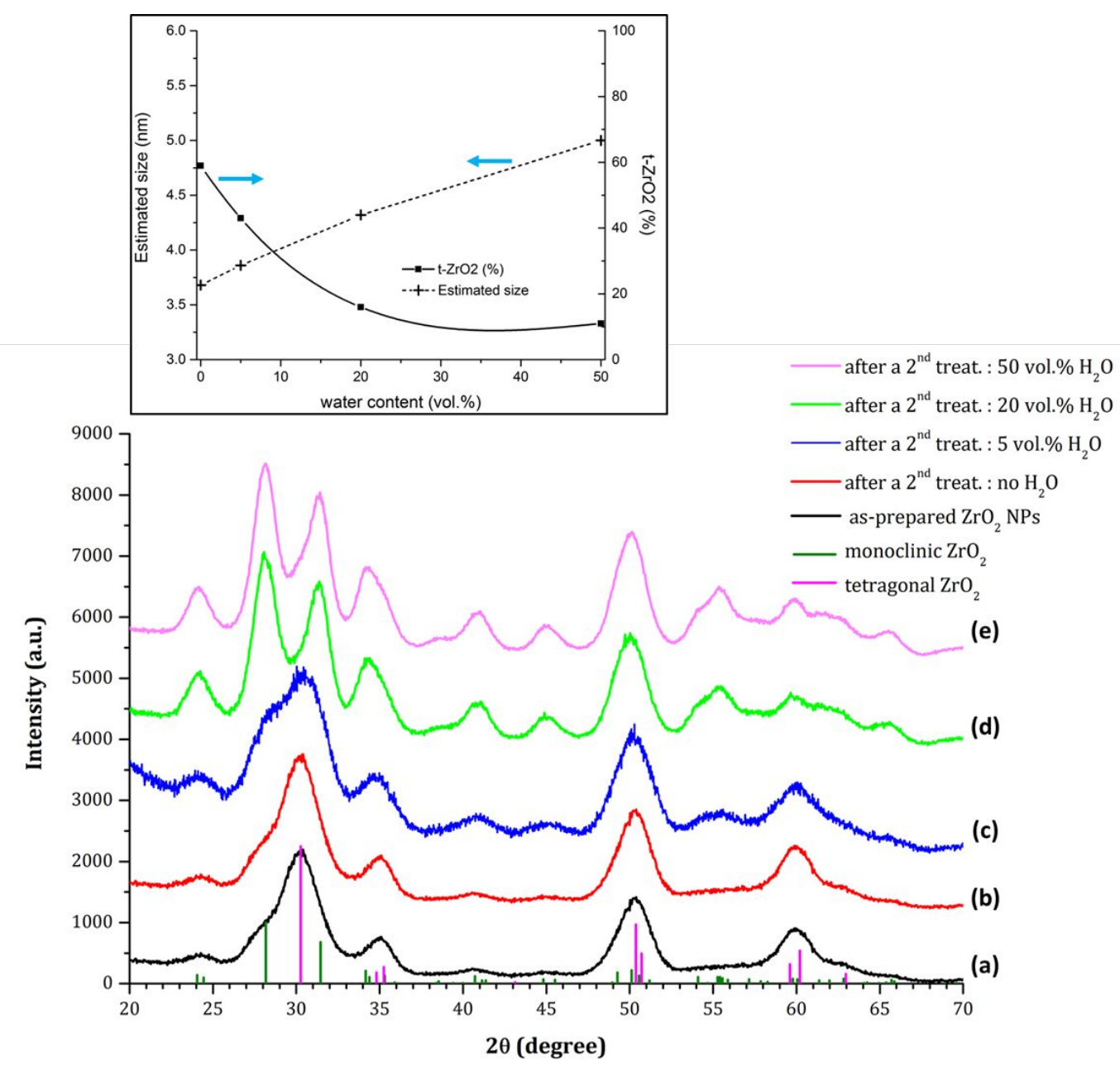

Figure.4. X-ray diffraction patterns of $\mathrm{ZrO}_{2}$ nanoparticles after a solvothermal post-treatment in a mixture of benzyl alcohol and deionized water with volume ratios of $0,5,20$ and $50 \mathrm{vol} . \%$ (a-e). Also shown are the estimated size and $\mathrm{t}-\mathrm{ZrO}_{2}$ weight fraction (inset). 
Those results, i.e. the effect of the presence of water in the initial reaction mixture and/or effect of a subsequent solvothermal post-treatment in hydrated medium, suggest that the biphasic nature of the reference sample synthesized in anhydrous benzyl alcohol with a nominal zirconium isopropoxide concentration of $0.32 \mathrm{mol.L}-1$ is essentially due to the side dehydration reaction of isopropanol and to the release of water during the solvothermal treatment. We evidenced a water-induced phase conversion of the $\mathrm{t}-\mathrm{ZrO}_{2}$ into $\mathrm{m}-\mathrm{ZrO}_{2}$ nanoparticles which overpasses the stabilization offered by the critical size effect evidenced by Garvie. Xie et al. found that pure $\mathrm{t}-\mathrm{ZrO}_{2}$ nanocrystals exhibiting large surface area underwent a tetragonal-tomonoclinic transformation of about $78 \%$ after 12 hours of immersion in liquid water or aqueous vapor at $25^{\circ} \mathrm{C} .{ }^{8}$ Moreover, the transformation was not accompanied by any particle growth or surface area change. From these observations, they attributed the origin of this polymorphic transformation to a decrease in the driving force initially responsible for the room temperature stabilization of $\mathrm{t}-\mathrm{ZrO}_{2}$. They concluded that under water exposure, the hydration of the nanoparticle surface leads to a significant decrease of the difference in surface free energy between the two polymorphs and thus to a strong decrease of the critical size.

Such a transformation was probably not detected by Garnweitner et al. and De Keukeleere et al. because they synthesized their samples at lower zirconium precursor concentrations than those selected in this study and especially for De Keukeleere et al. with a fast and effective microwave solvothermal treatment. ${ }^{25,35,38} \mathrm{We}$ can conclude that the water-induced phase conversion is clearly dependent on the water content of the reaction mixture but also on the reaction time of the solvothermal treatment since some organic ligands cover the nanoparticles surface and slow down the process. The largest fraction of $\mathrm{m}-\mathrm{ZrO}_{2}$ nanoparticles evidenced in the sample prepared with the highest zirconium alkoxide concentration of 1.28 mol.L $\mathrm{L}^{-1}$ could be explained by: (i) the formation of a large number of nanoparticles per volume unit, (ii) the 
development of a large surface capable of catalyzing isopropanol dehydration, (iii) the release of a large content of water and thus, (iv) the extensive tetragonal-to-monoclinic transformation induced by the highest water content.

It is well known to date that depending on its crystalline polymorphs, zirconia can be used in numerous applications like oxygen sensors, gate dielectrics and bioactive coatings. For example, the catalytic conversion of syngas will lead either to ethanol or to isobutanol depending on whether they are tetragonal or monoclinic polymorphs. It is therefore crucial to be able to obtain single phase and crystalline nanoparticles. Whereas De Keukeleere and coworkers have developed a fast and tunable synthesis of $\mathrm{ZrO}_{2}$ nanocrystals limiting the eventuality of water-induced phase conversion, we propose another strategy which enables the production of pure $\mathrm{t}-\mathrm{ZrO}_{2}$ nanoparticles whatever the precursor and its concentration selected for the synthesis.

\section{III.2. Water-free production of $\mathrm{t}-\mathrm{ZrO}_{2}$ nanocrystals}

In order to avoid the detrimental effect of water on the phase purity of nanocrystals synthesized by the benzyl alcohol route, we decided to introduce a sodium source in the form of sodium metal. Indeed, it was found in the literature that alkali cations covering zirconia catalyst are susceptible to inhibit zirconia-catalyzed alcohol dehydration. ${ }^{42}$ Moreover, sodium alkoxides issued from the dissolution of sodium metal are able to neutralize traces of water and give another chance to limit and to trap any water molecules that may appear during the synthesis. This method firstly proposed by Kyrides et al. has proven to be a suitable procedure for dehydration of an alcoholic medium. ${ }^{43}$ The reactions occurring during the process are represented as follows:

$$
\mathrm{Na}_{(s)}+\mathrm{ROH}_{(\ell)} \rightarrow \mathrm{NaOR}_{(\mathrm{solv})}+\frac{1}{2} \mathrm{H}_{2}(\mathrm{~g})
$$




$$
\mathrm{NaOR}_{(\text {solv })}+\mathrm{H}_{2} \mathrm{O}_{(\text {solv })} \rightleftarrows \mathrm{NaOH}_{(\text {solv })}+\mathrm{ROH}_{(\text {solv })}
$$

The experimental protocol was simply modified by including a preliminary step of sodium metal dissolution in benzyl alcohol at room temperature for at least 30 minutes. This transparent sodium benzoxide solution is then used for the rest of the synthesis conformingly to the protocol exposed in the experimental section.

\section{a) Effect of sodium-to-zirconium molar ration on sample purity}

In this part, the influence of the nominal sodium content used to generate the sodium benzoxide species is first studied in the case of a zirconium isopropoxide concentration of 0.32 mol.L-1 and then developed for extremely high zirconium isopropoxide concentration of 1.28 mol.L $\mathrm{L}^{-1}$ in order to illustrate the exceptional efficiency of the proposed method.

In the case of a zirconium isopropoxide concentration of $0.32 \mathrm{~mol} . \mathrm{L}^{-1}$, we found a strong dependence of the sample purity by varying the content of sodium metal from $0,0.05,0.10$, 0.20, 0.40 and 1.0 mol. $\mathrm{L}^{-1}$ as it is shown in the Figure 5. Up to a concentration of $0.10 \mathrm{~mol} . \mathrm{L}^{-1}$ in sodium, the presence of $\mathrm{m}-\mathrm{ZrO}_{2}$ polymorph is still discernable but tends to vanish since the fraction of $\mathrm{m}-\mathrm{ZrO}_{2}$ decreases from $44 \mathrm{wt} . \%$ to $40 \mathrm{wt} . \%$ for a sodium concentration of 0.05 mol.L $\mathrm{L}^{-1}$ and to $23 \mathrm{wt} . \%$ for a sodium concentration of $0.10 \mathrm{~mol} . \mathrm{L}^{-1}$. From 0.20 to $0.40 \mathrm{~mol} . \mathrm{L}^{-1}$, the final sample is only characterized by the presence of the $\mathrm{t}-\mathrm{ZrO}_{2}$ phase while a secondary phase appears for a high molar ratio in sodium of $1.0 \mathrm{~mol} . \mathrm{L}^{-1}$. It is important to indicate that the content of sodium benzoxide species strongly impacts the purity of the final zirconia sample but also promotes the crystalline growth. Indeed, the particle size is $3.2 \mathrm{~nm}$ for a sodium concentration of $0.20 \mathrm{~mol} . \mathrm{L}^{-1}$ and reaches $4.8 \mathrm{~nm}$ when a sodium concentration of 0.40 mol. $\mathrm{L}^{-}$ 1 is applied. At last, an excessive addition of sodium causes the appearance of an extra compound attributed to sodium zirconate, $\mathrm{Na}_{2} \mathrm{ZrO}_{3}$ jointly to the narrowing of the Bragg 
reflections of the zirconia nanoparticles indicating that the reaction mixture and especially the by-products have an activated action on the particle growth.

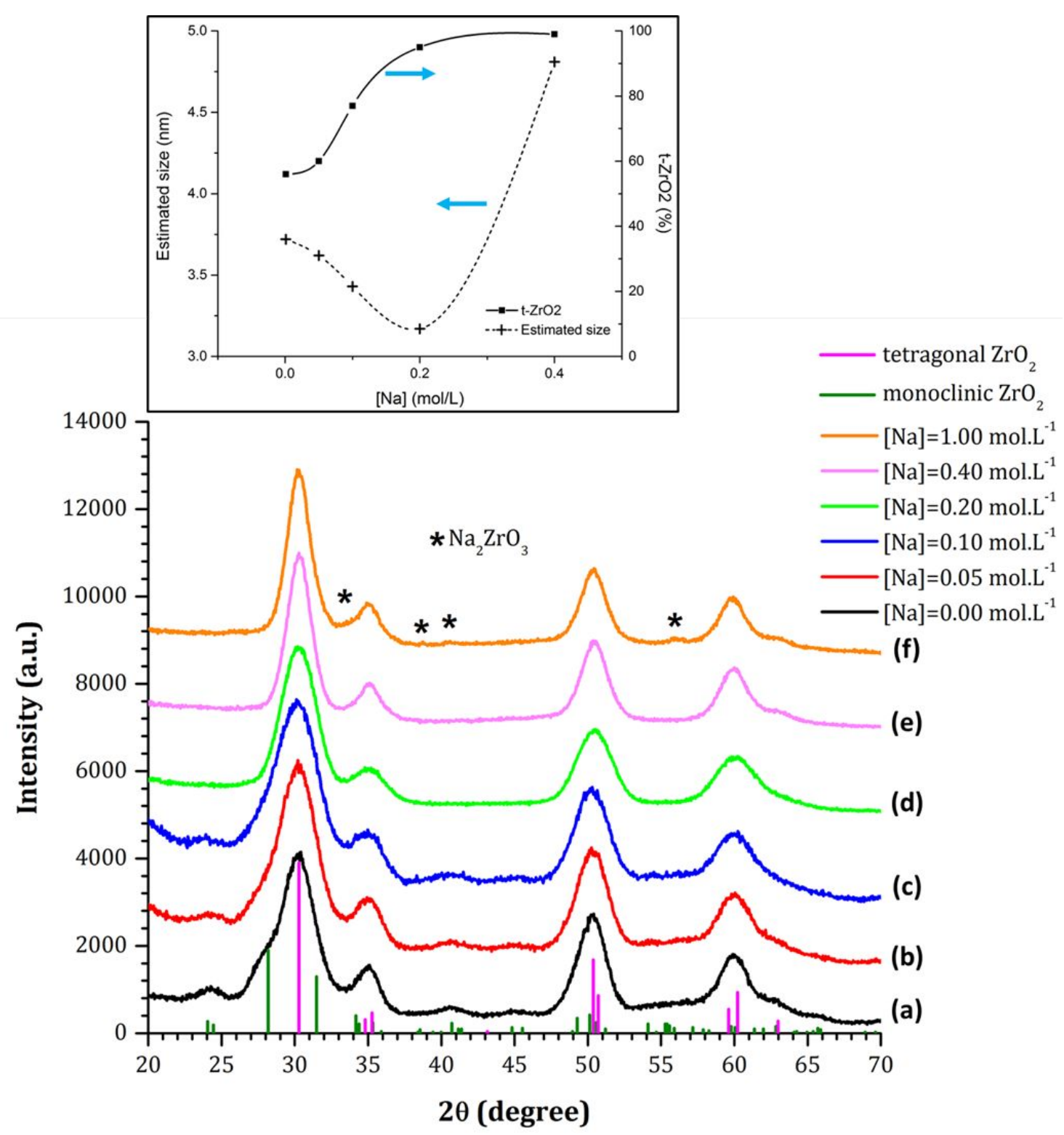

Figure.5. X-ray diffraction patterns of $\mathrm{ZrO}_{2}$ nanoparticles prepared with $[\mathrm{Zr}]=0.32$ mol.L $\mathrm{L}^{-1}$ by the Na-based benzyl alcohol sol-gel route as a function of the $\mathrm{Na}$ content $\left(\mathrm{mol}_{\mathrm{L}} \mathrm{L}^{-1}\right)$ in the reaction mixture. The stars underline the characteristic peaks of $\mathrm{Na}_{2} \mathrm{ZrO}_{3}$ signature. Also shown are the estimated size and $\mathrm{t}-\mathrm{ZrO}_{2}$ weight fraction (inset).

From 0.10 up to 0.40 mol. $\mathrm{L}^{-1}$, the samples are single phased and one can observe together with the disappearance of the monoclinic polymorph a slight decrease in the peak broadening of $\mathrm{t}-\mathrm{ZrO}_{2}$ indicating that the best compromise between the polymorphic selection and the grain 
growth limitation seems to be a concentration of 0.20 mol. $\mathrm{L}^{-1}$ in sodium metal for a zirconium isopropoxide concentration of $0.32 \mathrm{~mol} . \mathrm{L}^{-1}$. For this sample, the Rietveld refinement of the XRD diagram using the tetragonal symmetry gives an apparent size of $3.51 \mathrm{~nm}$.

Another series of experiments was conducted in order to confirm the efficiency of the proposed experimental strategy to promote a good phase purity whatever the precursor content. The concentration in zirconium isopropoxide was increased by a factor 4 up to $1.28 \mathrm{~mol} . \mathrm{L}^{-1}$ with two different concentrations of sodium metal of 0.20 mol.L $\mathrm{L}^{-1}$ and 0.80 mol.L-1 corresponding to a sodium-to-zirconium molar ratio of around 0.15 and 0.62 respectively. The molar ratio of 0.62 corresponds to the sodium-to-zirconium ratio used in the case of the optimized sample prepared with zirconium isopropoxide at $0.32 \mathrm{~mol} . \mathrm{L}^{-1}$ and sodium metal at 0.20 mol. $\mathrm{L}^{-1}$ whereas the molar ratio of 0.15 corresponds to the sodium-to-zirconium ratio used in the case of the sample prepared with zirconium isopropoxide at $0.32 \mathrm{~mol}^{-\mathrm{L}^{-1}}$ and sodium metal at 0.05 mol.L-1.

The XRD diagrams of the resulting powders are gathered in the Figure 6. At first sight, the content of monoclinic phase seems to be dependent on the concentration of zirconium alkoxide and then on the concentration of isopropanol that can be released upon the dissolution of the precursor and the ligand exchange reaction. In the absence of alkaline species, a larger concentration of the isopropanol adduct leads to a larger content of monoclinic nanocrystals which confirms that the isopropanol dehydration is the predominant side-reaction responsible for the proportional phase conversion process. At high concentration in zirconium alkoxide, it is not possible to recover a single-phase sample by the use of sodium in $0.20 \mathrm{~mol} . \mathrm{L}^{-1}$ concentration as it was the case for the optimized Na-based $\mathrm{t}-\mathrm{ZrO}_{2}$ sample but the Figure 6 shows that the recovering of pure $\mathrm{t}-\mathrm{ZrO}_{2}$ nanocrystals is possible by using a larger concentration of sodium of $0.80 \mathrm{~mol} . \mathrm{L}^{-1}$. Such a concentration corresponds to a sodium-to-zirconium molar ratio of 0.62 , similar to those that has been used in the optimized conditions for the Na-based 
benzyl alcohol route, and shows that a minimal sodium-to-zirconium molar ratio comprised between 0.31 and 0.62 is necessary to counterbalance the effect of the release of in-situ water.

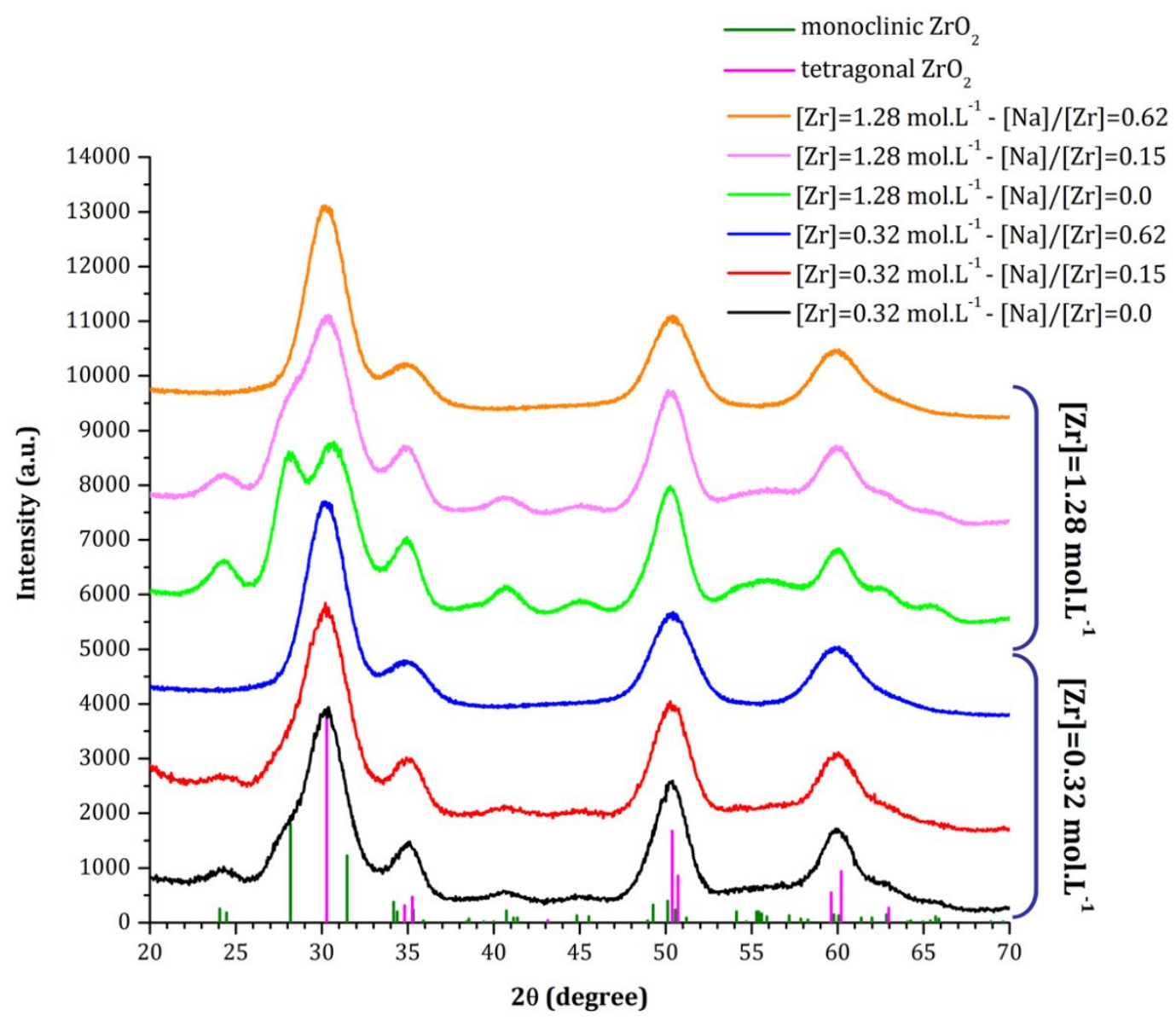

Figure.6. X-ray diffraction patterns of $\mathrm{ZrO}_{2}$ nanoparticles prepared by the benzyl alcohol route for two zirconium isopropoxide concentrations $\left(0.32\right.$ or 1.28 mol. $\left.\mathrm{L}^{-1}\right)$ w/out sodium introduced at a $[\mathrm{Na}] /[\mathrm{Zr}]$ ratio of $0,0.15$ or 0.62 .

The crystallinity and morphology of the selected Na-based sample $\left([\mathrm{Zr}]=0.32\right.$ mol.L $\mathrm{L}^{-1}$ and $[\mathrm{Na}]=0.20 \mathrm{~mol} . \mathrm{L}^{-1}$ ) were also confirmed by the TEM study displayed in the Figure 7 . In the case of the washed and wet particles (Figure 7(a)), an overview image of the dispersion of nanoparticles on the TEM grid at low magnification illustrates that the sample prepared from the wet particles entirely consists of nanosized and monodisperse objects whereas the SAED analysis of the Figure 7(b) reveals some continuous and slightly diffuse diffraction rings corresponding to the tetragonal or cubic polymorph of zirconia. The last two images (Figure 
$7(\mathrm{c}-\mathrm{d}))$ illustrate the isolated nature of the crystallized nanoparticles measuring around $3.0 \mathrm{~nm}$ whatever the way of TEM grid preparation by a wet or a dry process. The particles exhibit generally a more pronounced faceted-shape than the particles issued from the Na-free benzyl alcohol route. The alkaline medium and/or a possible change in the reaction pathway could be evocated in order to explain the more pronounced expression of crystallinity and the tailoring of faceted nanocrystals. Alkaline organic base or resulting hydroxide species after neutralization of water play a role of mineralizing agent favoring the solubility of surface ions imperfectly placed in the network and resulting in the exchange of these ions by others in order to achieve a crystal that would be gradually perfect.

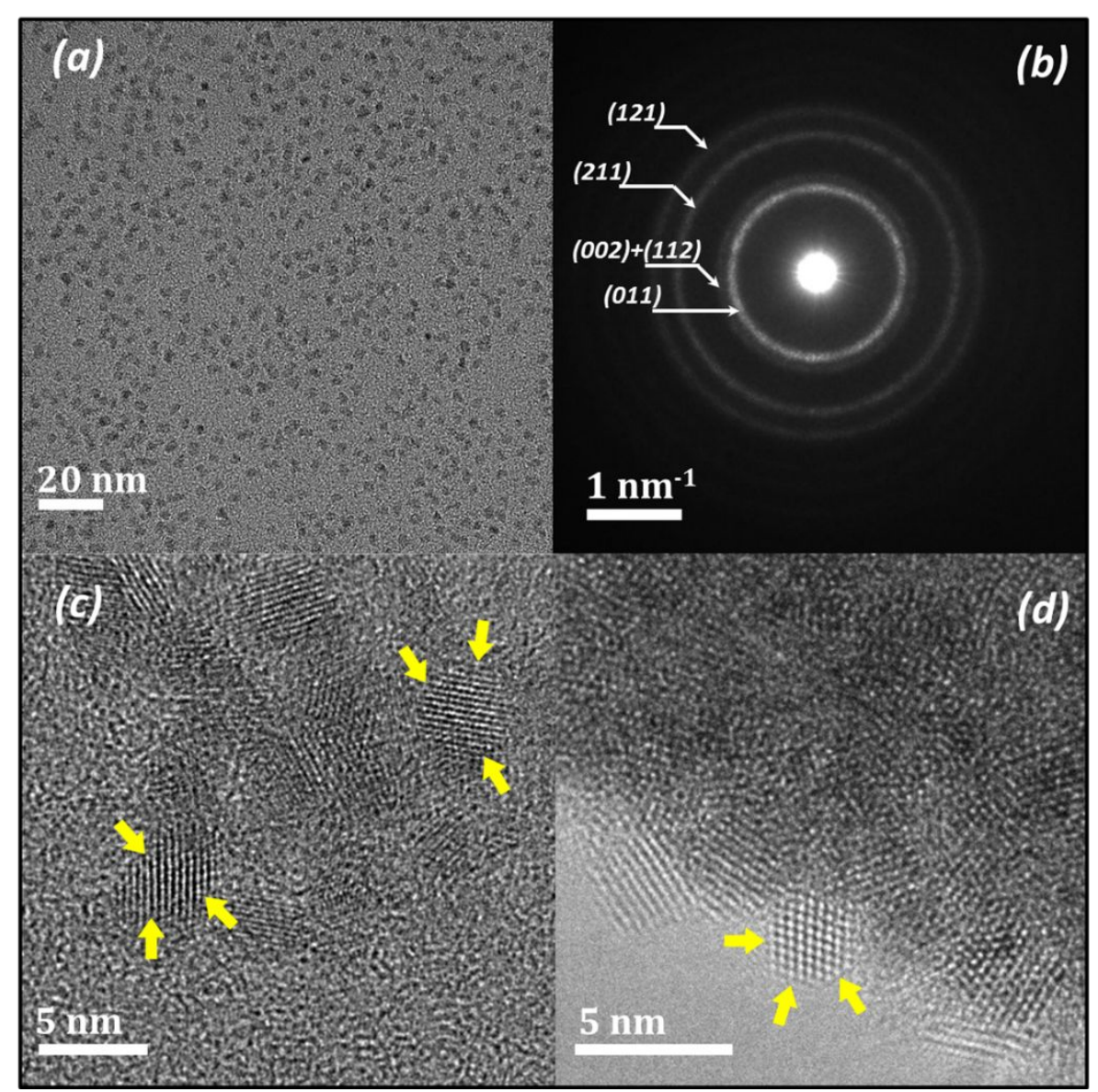

Figure. 7. TEM micrographs of $\mathrm{ZrO}_{2}$ nanoparticles prepared by the Na-based benzyl alcohol route $\left([\mathrm{Zr}]=0.32 \mathrm{~mol} . \mathrm{L}^{-1}\right.$ and $\left.[\mathrm{Na}]=0.20 \mathrm{~mol} \cdot \mathrm{L}^{-1}\right)$ : (a) image of wet particles at low magnification, (b) diffraction rings of the corresponding wet particles, (c) and (d) images of dried particles. 
According to the literature, under alkaline reaction conditions or in some rare cases in the presence of metal precursors exhibiting a strong Lewis acidity, the reaction between metal alkoxides and alcohols undergoes a Guerbet-type C-C coupling rather than an ether elimination. ${ }^{34,36-37,42}$ To investigate a possible change in the condensation reaction, the supernatant liquid collected after centrifugation was subjected to ${ }^{13} \mathrm{C}$ and ${ }^{1} \mathrm{H}$ NMR analyses revealing the presence of the initial benzyl alcohol, isopropanol, minor presence of 4-phenyl-2-butanol ( $\beta$-ramified Guerbet alcohol) and the absence of water (Figure.S2. in supporting information).

Since only minor quantity of 4-phenyl-2-butanol is found into the supernatant, the mechanism for the formation of M-O-M network is thus possibly based on C-C coupling and/or a direct hydroxylation of the metal alkoxide by $\mathrm{NaOH}$ which is a byproduct of the neutralization of water by sodium benzoxide. ${ }^{38}$ The Guerbet mechanism occurs via a complex sequence in several steps; namely, (i) dehydrogenation or oxidation of the alcohol to aldehyde, (ii) aldol condensation of aldehyde and enolate, (iii) dehydration of the aldol product, and, finally (iv) hydrogenation of the $\alpha, \beta$-unsaturated ketone giving the Guerbet alcohol. According to the work of Kozlowski, even if the alkali metal activates slightly the dehydrogenation reaction of alcohol, sodium cations tend to disrupt the appropriate acid-base pairs on the surface of zirconia, inhibit the aldol condensation step and then limit the possibility for the chemical system to generate Guerbet alcohols. ${ }^{42}$

Finally, the addition of sodium metal to the benzyl alcohol at a concentration of 0.20 mol.L $\mathrm{L}^{-1}$ succeeded for a zirconium isopropoxide concentration of $0.32 \mathrm{~mol} . \mathrm{L}^{-1}$ to eliminate the traces of water. Concerning the absence of water into the supernatant, two hypotheses or a combination of both can be proposed: either based on the proposition of Kyrides the acid-base neutralization of water occurs owing to the sodium benzoxide species and/or as it was proposed by Kozlowski 
the inhibition of isopropanol dehydration onto zirconia nanoparticles is due to $\mathrm{Na}^{+}$pollution all over the surface..$^{42-43}$

\section{b) Complementary experiments: localization of $\mathrm{Na}$}

To investigate how the modification of the benzyl alcohol route by the addition of sodium metal is acting and what specificities have to be taken in account, we have performed complementary experiments, namely ICP-AES, thermal analyses, and, FTIR spectroscopy.

According to the ICP-AES measurements, a weight fraction of approximately 0.08 in sodium has been measured in the sample prepared with the following conditions $[\mathrm{Zr}]=0.32 \mathrm{~mol} . \mathrm{L}^{-1}$ and $[\mathrm{Na}]=0.20 \mathrm{~mol} \cdot \mathrm{L}^{-1}$. Although the weight fraction value is quite large, it could be explained by taking into account the large developed specific surface commonly found in the case of nanoparticles compared to those observed for larger micrometric systems. Even if the cations are supposed to be used for the surface covering of the particles, it is not excluded that a $\mathrm{Na}^{+}$-doping phenomenon occurs and stabilizes the $\mathrm{t}-\mathrm{ZrO} 2$ phase. For both cases, $\mathrm{Na}$-free or Namodified benzyl alcohol route, powders were submitted to thermal gravimetric analyses. Displayed in the Figure 8 the thermal gravimetric curves exhibit the same global feature composed of two main weight losses. The first weight loss is generally attributed to the evaporation of solvents and physically adsorbed molecules. From room temperature to $400^{\circ} \mathrm{C}$, the weight loss is of about $8.4 \%$ for the sodium-free sample and $10.2 \%$ for the sodium-based sample. The second weight loss is detectable around $440^{\circ} \mathrm{C}$ for the sodium-free sample and estimated to 5.5\%; such feature is usually attributed to the release of chemisorbed molecules, to the dehydration of hydroxyl and alkoxy groups and of course to the combustion of residual organics. In the case of the sodium-based synthesis, the second weight loss is shifted up to $650^{\circ} \mathrm{C}$ and supposed to correspond to the decarbonation of a refractory intermediate compound, 
possibly $\mathrm{Na}_{2} \mathrm{CO}_{3}$ which shall decompose at higher temperature around $650^{\circ} \mathrm{C}-750^{\circ} \mathrm{C}$. The formation of sodium carbonate could occur at the surface of the nanoparticles due to air exposure at the end of the drying step, after the full removal of dichloromethane, followed by the acid-base reaction of carbon dioxide onto the surface of the particles. In order to support this hypothesis, FTIR analysis has been undertaken and the presence of carboxylate or carbonate species was evidenced in the dried powder. Indeed, the FTIR spectrum of the modified Na-based benzyl alcohol route exhibits two bands at $1550 \mathrm{~cm}^{-1}$ and $1430 \mathrm{~cm}^{-1}$ that can be attributed to the vibrational band of the carbonyl function of a carboxylate or carbonate group (Figure.S3. in supporting information).
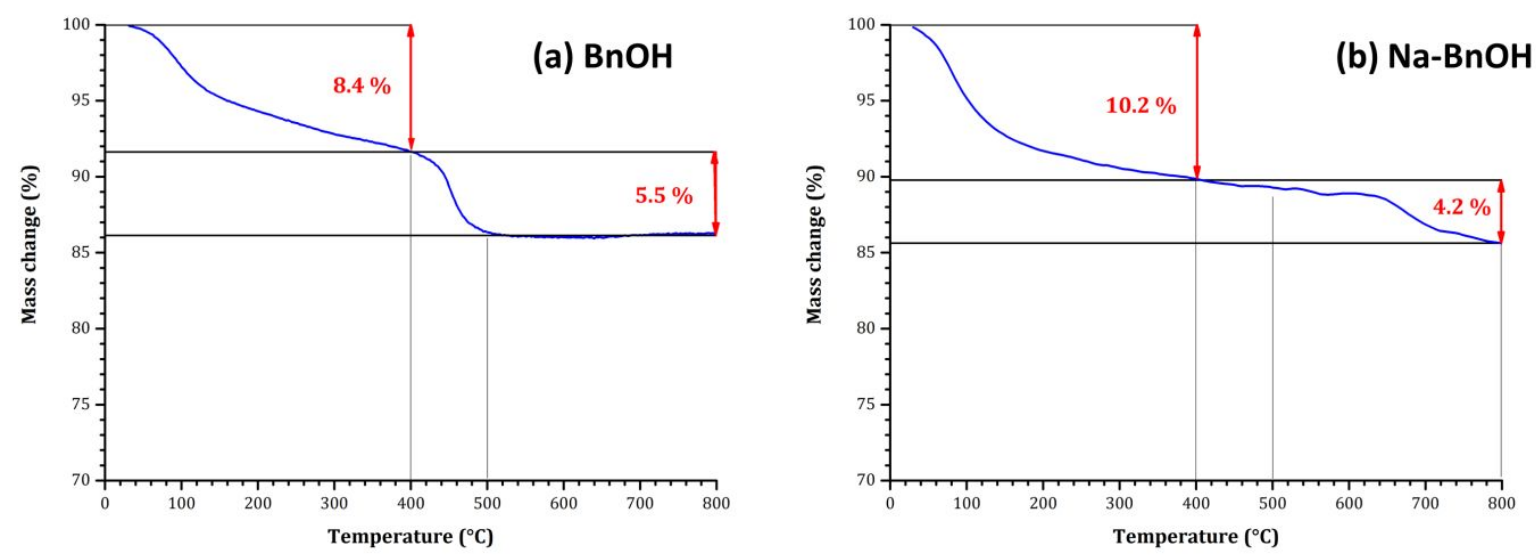

Figure.8. TG curves of $\mathrm{ZrO}_{2}$ nanoparticles obtained (a) by a Na-free procedure and (b) by a Na-based procedure in air $\left(10^{\circ} \mathrm{C} \cdot \mathrm{min}^{-1}\right)$.

Even if the results indicate that the largest part of the sodium is located at the surface of the nanoparticles where it forms a carbonate compound, a possible $\mathrm{Na}^{+}$-doping phenomenon is not discarded. To highlight this questionable aspect we examine if the use of different sodium sources for the Na-modified benzyl alcohol route leads also to the stabilization of $\mathrm{t}-\mathrm{ZrO}_{2}$ nanoparticles. The last series of syntheses consisted to use and to compare the effect of different sodium sources, i.e. sodium metal $(\mathrm{Na})$, sodium isopropoxide $\left(\mathrm{NaO}^{\mathrm{i}} \mathrm{Pr}\right)$ and lastly sodium chloride $(\mathrm{NaCl})$ maintaining all the other parameters $\left(210^{\circ} \mathrm{C}, 48\right.$ hours and $[\mathrm{Zr}]=0.32 \mathrm{~mol} . \mathrm{L}^{-1}$ 
and $[\mathrm{Na}$ source $\left.]=0.20 \mathrm{~mol} \cdot \mathrm{L}^{-1}\right)$. It is important to note that whereas sodium benzoxide or isopropoxide are some strong organic bases in nonaqueous amphiprotic medium like alcohols, chloride only expresses a weak Brønsted and borderline Lewis basicities. The XRD diagrams of the resulting samples are gathered in the Figure 9. By comparison with the Na-free sample, the Na- and the $\mathrm{NaO}$ Pr-based samples are exclusively composed of pure $\mathrm{t}-\mathrm{ZrO}_{2}$ nanoparticles whereas the NaCl-based sample remains definitively biphasic in nature. This last result demonstrates that the presence of $\mathrm{Na}^{+}$is not a sufficient factor to suppress the phase conversion of $\mathrm{t}-\mathrm{ZrO}_{2}$ into $\mathrm{m}-\mathrm{ZrO}_{2}$ phase contrary to what was observed in the Na-based- or NaOiPr-based benzyl alcohol syntheses.

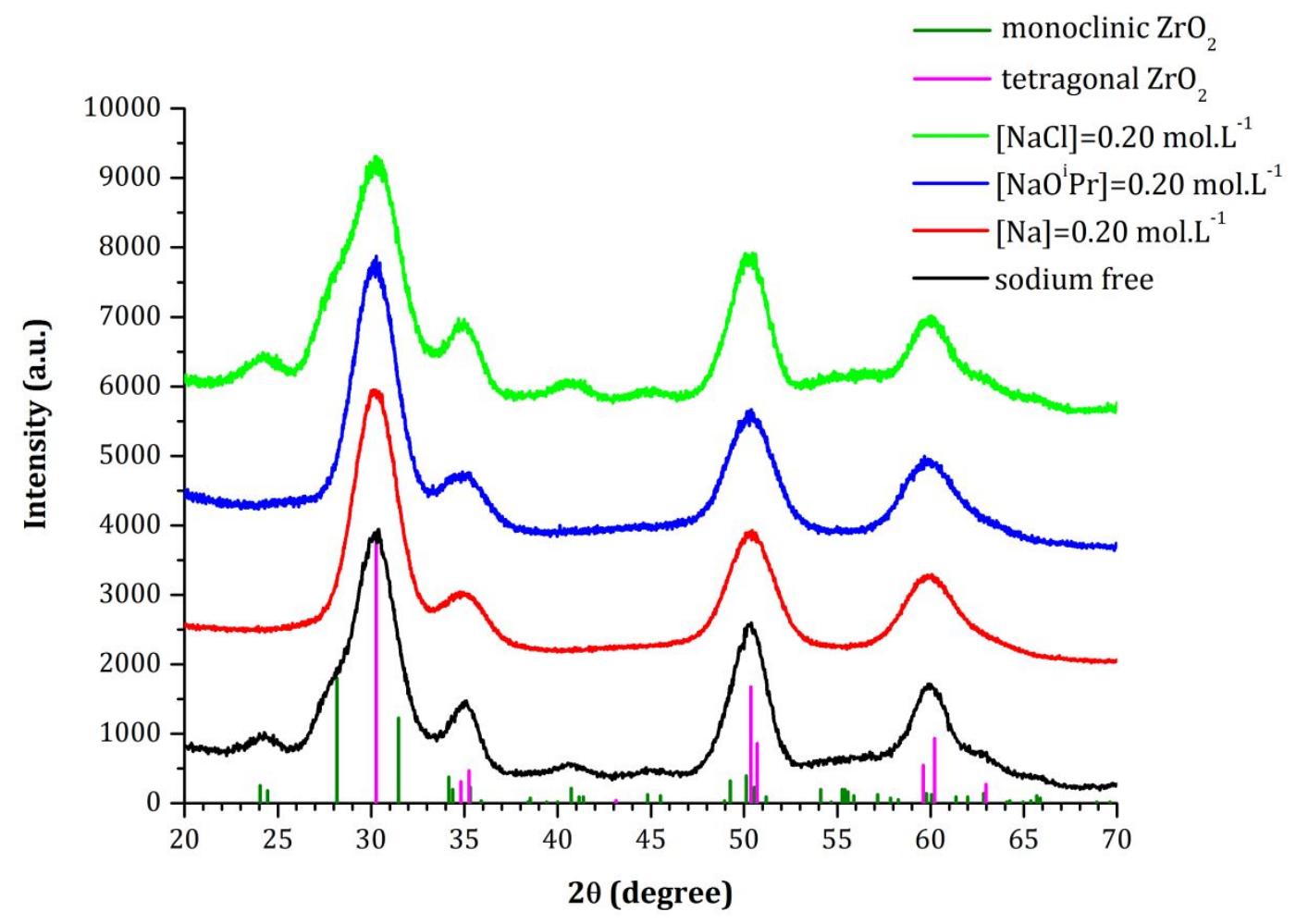

Figure.9. X-ray diffraction patterns of $\mathrm{ZrO}_{2}$ nanoparticles prepared by the benzyl alcohol route w/out sodium-based reactant $(\mathrm{Na}, \mathrm{NaO}$ Pr or $\mathrm{NaCl})$ introduced at 0.2 mol. $\mathrm{L}^{-1}$.

It gives also an indirect evidence that the phase selection mechanism does not operate via a $\mathrm{Na}^{+}$-doping effect but much more likely via a complex interplay of at least two main factors, 
i.e. the strong alkalinity of the anionic species appearing during the process and in a lesser extent the cationic capping of the nanoparticle surface inducing acid-base sites disruption.

\section{III.3. Discussion}

While the standard benzyl alcohol route of zirconium isopropoxide-isopropanol adduct based on an ether-elimination reaction leads to a biphasic mixture, the dissolution of sodium metal into anhydrous benzyl alcohol prior to the solvothermal treatment definitively plays a role of primary importance in order to obtain single-phase tetragonal nanocrystals. One important fact is that there was no difference in the average size estimated by Rietveld refinement or by TEM observation between both $\mathrm{t}$ - and $\mathrm{m}-\mathrm{ZrO}_{2}$ nanocrystals which means that no size effect seems to govern the polymorph conversion and the synthesis of a biphasic sample.

The progressive disappearance of the monoclinic contribution into the samples was observed as to follow that of the increase in the sodium metal content or more precisely the increase in the sodium benzoxide content. Beyond a certain amount comprised between the molar concentrations of 0.10 and $0.20 \mathrm{~mol} . \mathrm{L}^{-1}$ in sodium benzoxide, the content is large enough to neutralize the water appearing during the solvothermal treatment or to simply inhibit NP surface-catalyzed alcohol dehydration. The range of concentration corresponds to a sodium-tozirconium molar ratio between 0.31 and 0.62 with which the final mixture is found to be exclusively composed by some single-phase $\mathrm{t}-\mathrm{ZrO}_{2}$ nanocrystals without any excessive particle growth.

Two synthesis schemes for both benzyl alcohol and sodium-modified benzyl alcohol routes are proposed in the Figure 10. 

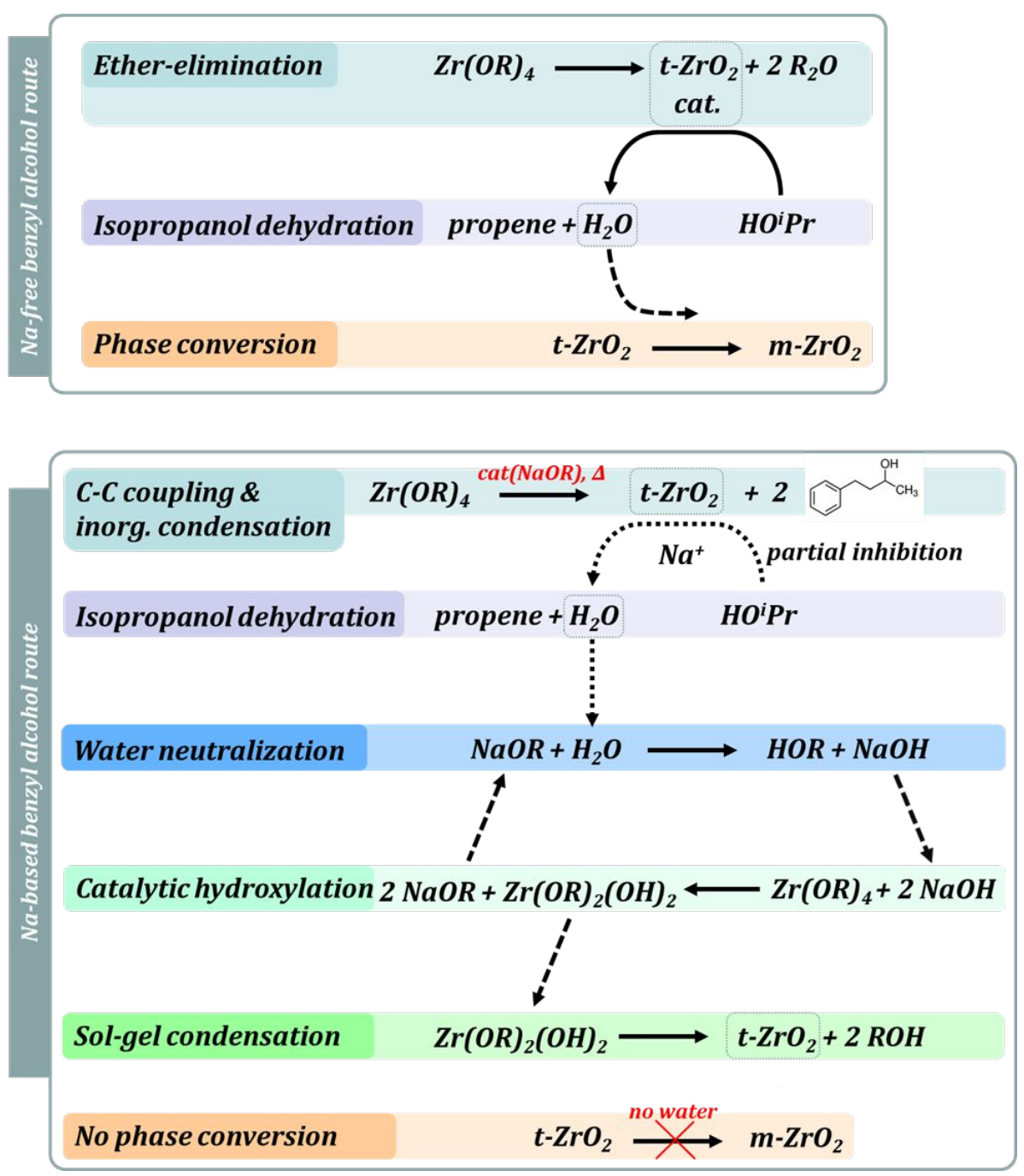

Figure.10. Summary of the main reactions involved in the Na-free and Na-based benzyl alcohol routes.

The emergence of the $\mathrm{m}-\mathrm{ZrO}_{2}$ nanocrystals in the case of the synthesis without or with only a low sodium content was attributed to the detrimental presence of in-situ water issued from a side-reaction as it is summarized in the top of the Figure 10. Such water is presumably released from a surface-mediated dehydration mechanism of the alcoholic medium due to the amphoteric, and especially acidic, properties of zirconia nanocrystals. More precisely, the correlation between the $\mathrm{m}-\mathrm{ZrO}_{2}$ content and the concentration of the zirconium isopropoxideisopropanol adduct let us to think that the dehydration reaction essentially concerns the isopropanol molecules coming from the zirconium precursor or issued from the alcohol 
exchange reaction with benzyl alcohol and leads to the release of volatile propene and water. The intentional addition of water into the reaction vessel as well as a post-synthesis retreatment of the freshly as-prepared particles in the presence of water has shown that the water degraded the purity of the samples and induced an effective phase conversion process proportional to the water content introduced into the reactor. Such observations have already been reported by Murase et al. who evidenced the transformation of metastable tetragonal zirconia into monoclinic one upon thermal treatment or contact with water vapor at $400^{\circ} \mathrm{C} .{ }^{12} \mathrm{Xie}$ et al. confirmed the same feature by in situ Raman spectroscopy in the case of calcined zirconium oxyhydroxide which leads to tetragonal zirconia upon heat-treatment at $350^{\circ} \mathrm{C}$ and then transforms into monoclinic zirconia without any drastic loss in surface area by the simple exposure to water vapor even at room temperature. ${ }^{8}$ Since the tetragonal-to-monoclinic transformation is described as a martensitic and diffusion-less process, there is no activation barrier for the process to occur and the only limitation lies in the formation of nucleation sites. We presume that such sites nucleate once the nanoparticles surface is depassivated, which means extensively hydrolyzed to remove organic ligands. The initiation of the water-induced phase conversion could be similar to the low thermal degradation observed for micrometric zirconia but is still unclear. ${ }^{44}$

The role played by the sodium alkoxide during the particle formation and the ageing of the suspension was ascertained by the substitution of $\mathrm{Na}$ metal with $\mathrm{NaO}$ i Pr and $\mathrm{NaCl}$ (even sodium hydroxide can be used but the result is not shown here). Taking in account that the $\mathrm{Na}^{+}$cations covering the surface of the zirconia nanoparticles are only partially inhibiting the dehydration side-reaction, we suggest that the strong alkalinity of the reaction mixture enables the sol-gel process to pursue in a non-hydrolytic manner. In the bottom of the Figure 10, we propose a summary of the different roles of sodium alkoxide. First, sodium alkoxide is a prerequisite for the $\mathrm{C}-\mathrm{C}$ bond formation, the hydroxylation of the zirconium precursor and further the 
condensation into $\mathrm{ZrO}_{2}$ nanoparticles. Such reaction is validated but only in a limited extent. Indeed, $\mathrm{Na}^{+}$is known to partially inhibit the Guerbet-like mechanism which is corroborated by the minor presence of 4-phenyl-2-butanol at the end of the solvothermal treatment. Secondly, the presence of $\mathrm{Na}^{+}$is also known to decrease the catalytic activity of the NP surface toward alcohol dehydration by poisoning its surface. Anyway sodium alkoxide can neutralize traces of water and releases sodium hydroxide which, in turn, is able to promote the inorganic condensation via a catalytic hydroxylation reaction. Following the non-hydrolytic hydroxylation, sodium alkoxide is regenerated and the hydroxylated species can undergo condensation via an alternate and/or complementary sol-gel reactions. At last, parallel to the formation process, the absence of water prevents the $\mathrm{t}-\mathrm{ZrO}_{2}$ nanoparticles to convert into monoclinic ones.

Interestingly, it was shown that the Na-modified benzyl alcohol route gave excellent results in terms of purity and limited particles size providing that the sodium-to-zirconium molar ratio was adjusted to at least 0.62 . The possibility to increase significantly the concentration of the reactants is of crucial interest when the chemical process is based on expensive solvent or when it is necessary to consider some industrial-scale or semi-industrial-scale developments.

The surface of the nanoparticles would require further investigations in terms of properties especially to determine the impact of the presence of sodium-based species poisoning the surface.

\section{CONCLUSION}

In conclusion, the tremendous effect of the presence of water on the growth and destabilization of $\mathrm{t}-\mathrm{ZrO}_{2}$ produced via the benzyl alcohol route was evidenced experimentally. Such a water, either intentionally added in the reaction mixture or issued from the dehydration of alcohol during a prolonged heat treatment (and specially a high zirconium alkoxide 
concentration), was shown to play a detrimental role similar to what was observed in the low thermal degradation of bulk zirconia. In order to remedy the deterioration of the sample purity, we investigated the use of different sodium sources, namely sodium metal, sodium chloride or sodium isopropoxide, and found that the crystalline phase was affected if the sodium source was able to exhibit a strong basicity. Indeed, the Brønsted acid-base consumption of water by sodium alkoxide coupled with a $\mathrm{Na}^{+}$capping of the nanoparticle surface was found to be necessary to inhibit the isopropanol dehydration and/or to neutralize the in-situ water without promoting an excessive particle growth.

The addition of a basic salt has been found to be a suitable strategy able to avoid the loss of phase purity and to recover the phase stabilization of $\mathrm{t}-\mathrm{ZrO}_{2}$ nanoparticles offered by the critical size effect. This strategy was shown to be effective even when the dehydration side-reaction responsible for the release of water is promoted by a large increase of the alkoxide concentration and/or by a prolonged heat-treatment. Such a situation is generally encountered due to the reduction of the economic cost of a process, i.e. for example by decreasing the content of anhydrous benzyl alcohol and/or by choosing a less expensive zirconium alkoxide as zirconium n-propoxide even if the parent alcohol is quite more sensitive to dehydration upon prolonged solvothermal treatment than isopropanol itself.

\section{ASSOCIATED CONTENT}

\section{Supporting Information}

NMR analyses of the sodium-free synthesis $\left([\mathrm{Zr}]=0.32 \mathrm{~mol} \cdot \mathrm{L}^{-1}, 210^{\circ} \mathrm{C}\right.$ for 48 hours $)$, NMR analyses of the sodium-based synthesis $\left([\mathrm{Zr}]=0.32 \mathrm{~mol} \cdot \mathrm{L}^{-1},[\mathrm{Na}] /[\mathrm{Zr}]=0.62,210^{\circ} \mathrm{C}\right.$ for 48 hours), FTIR analysis of the zirconia nanoparticles prepared by the sodium-modified benzyl alcohol route $\left([\mathrm{Zr}]=0.32 \mathrm{~mol} . \mathrm{L}^{-1},[\mathrm{Na}] /[\mathrm{Zr}]=0.62,210^{\circ} \mathrm{C}\right.$ for 48 hours $)$. 


\title{
AUTHOR INFORMATION
}

\section{Corresponding Author}

E-mail: fabien.remondiere@unilim.fr

\section{Present addresses}

Jess Gambe: Department of Physics, College of Science and Mathematics, MSU-Iligan Institute of Technology, Iligan city, Philippines

\section{ACKNOWLEDGEMENTS}

We would like to thank Pierre Carles for TEM observations, Julie Cornette for FTIR spectroscopy, Sandra Blanchet for ICP-AES analyses and Yves Champavier for NMR recording.

\author{
ABBREVIATIONS \\ NPs, nanoparticles; $\mathrm{HO}^{\mathrm{i}} \mathrm{Pr}$, isopropanol; OiPr, isopropoxide; $\mathrm{BnOH}$, benzyl alcohol; $\mathrm{NaOBn}$, \\ sodium benzoxide.

\section{REFERENCES} \\ (1) Garvie, R. C.; Hanink, R. H. J.; Pascoe, R. T.; Ceramic steel?, Nature, 1975, 258, 703-704. \\ (2) Yan, R.; Ding, D.; Lin, B.; Liu, M.; Meng, G.; Liu, X.; Thin yttria-stabilized zirconia electrolyte and \\ transition layers fabricated by particle suspension spray, J. Power Sources, 2007, 164, 567-571.
}


(3) Murase, Y.; Kato, E.; Role of water vapor in crystallite growth and tetragonal-monoclinic phase transformation of $\mathrm{ZrO}_{2}$, J. Am. Ceram. Soc., 1983, 66, 196-200.

(4) Kohno, Y.; Tanaka, T.; Funabiki, T.; Yoshida, S.; Photoreduction of carbon dioxide with hydrogen over $\mathrm{ZrO}_{2}$, Chem. Commun., 1997, 841-842.

(5) Sayama, K.; Arakawa, H.; Photocatalytic decomposition of water and photocatalytic reduction of carbon dioxide over zirconia catalyst, J. Phys. Chem., 1993, 97, 531-533.

(6) Ho, S.-M.; On the structural chemistry of zirconium oxide, Mater. Sci. Eng., 1982, 54, 23-29.

(7) Reidy, C.-J.; Fleming, T.-J.; Hampshire, S.; Towler, M.-R.; Comparison of microwave and conventionally sintered yttria-doped zirconia ceramics, Int. J. Appl. Ceram. Technol., 2011, 8, 1475-1485.

(8) Bellido, J.-D.-A.; De Souza, J.-E.; M'Peko, J.-C.; Assaf, E.-M.; Effect of adding $\mathrm{CaO}_{\text {to }} \mathrm{ZrO}_{2}$ support on nickel catalyst activity in dry reforming of methane, Appl. Catal. A., 2009, 358, 215-223.

(9) Garvie, R. C.; The occurrence of metastable tetragonal zirconia as a crystallite size effect, J. Phys. Chem., 1965, 69, 1238-1243.

(10) Garvie, R. C.; Stabilization of the tetragonal structure in zirconia microcrystals, J. Phys. Chem., 1978, 82, 218-224.

(11) Shukla, S.; Seal, S. J.; Thermodynamic tetragonal phase stability in sol-gel derived nanodomains of pure zirconia, Phys. Chem. B, 2004, 108, 3395-3399.

(12) Xie, S.; Iglesia, E.; Bell, A. T.; Water-assisted tetragonal-to-monoclinic phase transformation of $\mathrm{ZrO}_{2}$ at low temperatures, Chem. Mater., 2000, 12(8), 2442-2447.

(13) Becker, J.; Hald, P.; Bremholm, M.; Pedersen, J. S.; Chevallier, J.; Iversen, S. B.; Iversen, B. B.; Critical size of crystalline $\mathrm{ZrO}_{2}$ nanoparticles synthesized in near- and supercritical water and supercritical isopropyl alcohol, ACS Nano, 2008, 2, 1058-1068.

(14) Rong, Y.; Meng, Q.; Zhang, Y.; Hsu, T. Y.; Phase stability and its intrinsic conditions in nanocrystalline materials, Mater. Sci. Eng., A, 2006, 438, 414-419. 
(15) Yamaguchi, T.; Sasaki, H.; Tanabe, K.; High selectivities of zirconium oxide catalyst for isomerization of 1-butene and dehydration of sec-butanol, Chem. Lett., 1973, 1017-1018.

(16) Davis, B. H.; Metal oxide analogue of metal alloy catalysts, Appl. Surf. Sci., 1984, 19, 200-217.

(17) Kozlowski, J. T.; Davis, R. J.; Sodium modification of zirconia catalysts for ethanol coupling to 1-butanol, J. Energy. Chem., 2013, 22, 58-64.

(18) Nakano, Y.; Yamaguchi, T.; Tanabe, K.; Hydrogenation of conjugated dienes over $\mathrm{ZrO}_{2}$ by $\mathrm{H}_{2}$ and cyclohexadiene, J. Catal., 1983, 80, 307-314.

(19) Lei, T.; Xu, J. C.; Tang, Y.; Hua, W. M.; Gao, Z.; New solid superacid catalysts for n-butane isomerization: $\gamma-\mathrm{Al}_{2} \mathrm{O}_{3}$ or $\mathrm{SiO}_{2}$ supported sulfated zirconia, Appl. Catal. A, 2000, 192, 181-188.

(20) Stichert, W.; Schüth, F.; Kuba, S; Knözinger, H.; Monoclinic and tetragonal high surface area sulfated zirconias in butane isomerization: CO adsorption and catalytic results, J. Catal., 2001, 198, 277-285.

(21) Morterra, C.; Cerrato, G.; Pinna, F.; Signoretto, M.; Crystal phase, spectral features, and catalytic activity of sulfate-doped zirconia systems, J. Catal., 1995, 157, 109-123.

(22) Zhao, N.; Pan, D.; Nie, W.; Ji, X.; Two-phase synthesis of shape-controlled colloidal zirconia nanocrystals and their characterization, J. Am. Chem. Soc., 2006, 128(31), 10118-10124.

(23) Joo, J.; Yu, T.; Kim, Y. W.; Park, H. M.; Wu, F.; Zhang, J. Z.; Hyeon, T.; Multigram scale synthesis and characterization of monodisperse tetragonal zirconia nanocrystals, J. Am. Chem. Soc., 2003, 125(21), 6553-6557.

(24) Inoue, M.; Kominami, H.; Inui, T; Novel synthetic method for the catalytic use of thermally stable zirconia: thermal decomposition of zirconium alkoxides in organic media, Appl. Catal. A, 1993, 97, L25-L30.

(25) Kongwudthiti, S.; Praserthdam, P.; Silveston, P.; Inoue, M.; Influence of synthesis conditions on the preparation of zirconia powder by the glycothermal method, Ceram. Int., 2003, 29, 807-814.

(26) Garnweitner, G.; Goldenberg, L. M.; Sakhno, O. V.; Antonietti, M.; Niederberger, M.; Stumpe, J.; Largescale synthesis of organophilic zirconia nanoparticles and their application in organic-inorganic nanocomposites for efficient volume holography, Small, 2007, 3(9), 1626-1632. 
(27) Zhou, S.; Garnweitner, G.; Niederberger, M.; Antonietti, M.; Dispersion behavior of zirconia nanocrystals and their surface functionalization with vinyl group-containing ligands, Langmuir, 2007, 23(18), 9178-9187.

(28) Niederberger, M.; Garnweitner, G.; Organic reaction pathways in the nonaqueous synthesis of metal oxide nanoparticles, Chem. Eur. J., 2006, 12, 7282-7302.

(29) Garnweitner, G.; Grote, C.; In situ investigation of molecular kinetics and particle formation of waterdispersible titania nanocrystals, Phys. Chem. Chem. Phys., 2009, 11(9), 3767-3774.

(30) Zhang, L.; Garnweitner, G.; Djerdj, I.; Antonietti, M.; Niederberger, M.; Generalized nonaqueous sol-gel synthesis of different transition-metal niobate nanocrystals and analysis of the growth mechanism, Chem. Asian $J .$, 2008, 3(4), 746-752.

(31) De Roo, J.; De Keukeleere, K.; Feys, J.; Lommens, P.; Hens, Z.; Van Driessche, I.; Fast, microwaveassisted synthesis of monodisperse $\mathrm{HfO}_{2}$ nanoparticles, J. Nanoparticle Res., 2013, 15(7).

(32) De Roo, J. ; Van Driessche, I. ; Martins, J.C. ; Hens, Z. ; Colloidal metal oxide nanocrystal catalysis by sustained chemically driven ligand displacement, Nat. Mater., 2016, 15(5), 517-521.

(33) Wang, J.; Cao, F.; Bian, Z.; Leung, M.K.H.; Li, H.; Ultrafine single-crystal TiOF 2 nanocubes with mesoporous structure, high activity and durability in visible light driven photocatalysis, Nanoscale, 2014, 6(2), 897-902.

(34) Niederberger, M.; Pinna, N.; Metal oxide nanoparticles in organic solvents, 2009, Springer-Verlag London.

(35) De Keukelere, K.; De Roo, J. ; Lommens, P. ; Martins, J.C. ; Van Der Voort, P. ; Van Driessche, I. ; Fast and tunable synthesis of $\mathrm{ZrO}_{2}$ nanocrystals: mechanistic insights into precursor dependence, Inorg. Chem. ; 2015, $54,3469-3476$

(36) Guerbet, M.; Action des alcools sur les dérivés sodés d'autres alcools, Compt. Rend., 1902, 135, $172-175$.

(37) Guerbet, M.; Sur trois alcools primaires nouveaux résultant de la condensation du benzylate de sodium avec les alcools propylique, butylique et isoamylique, Compt. Rend., 1908, 146, 1405-1407. 
(38) Garnweitner, G., Nonaqueous synthesis of transition-metal oxide nanoparticles and their formation mechanism (PhD dissertation), 2005, University of Potsdam.

(39) Kostestkyy, P.; Yu, J.; Gorte, R.J.; Mpourmpakis, G.; Structure-activity relationships on metal-oxides: Alcohol dehydration, Cat. Sci. \& Tech. ; 2014, 4(11), 3861-3869.

(40) Aramendia, M.A. ; Boráu, V. ; Jiménez, C. ; Marinas, J.M. ; Porras, A. ; Urbano, F.J. ; Synthesis and characterization of $\mathrm{ZrO}_{2}$ as an acid-base catalyst: dehydration-dehydrogenation of propan-2-ol, J. Chem. Soc., Faraday Trans., 1997, 93(7), 1431-1438.

(41) Haffad, D.; Chambellan, A.; Lavalley, J. C.; Propan-2-ol transformation on simple metal oxides $\mathrm{TiO}_{2}$, $\mathrm{ZrO}_{2}$ and $\mathrm{CeO}_{2}$, J. Mol. Catal. A, Chem., 2001, 168, 153-164.

(42) Kozlowski, J. T.; Davis, R. J.; Sodium modification of zirconia catalysts for ethanol coupling to 1-butanol, J. Energy. Chem., 2013, 22, 58-64.

(43) Kyrides, L.P.; Carswell, T.S.; Pfeifer, C.E.; Wobus, R.S.; Dehydration of alcohols with alkali metal alcoholates, Ind. Eng. Chem., 1932, 24(7), 795-797.

(44) Chevalier, J.; Gremillard, L.; Virkar, A.V.; Clarke, D.R.; The tetragonal-monoclinic transformation in zirconia: lessons learned and future trends, J. Am. Ceram. Soc., 2009, 92(9), 1901-1920. 


\section{For Table of Contents Only}

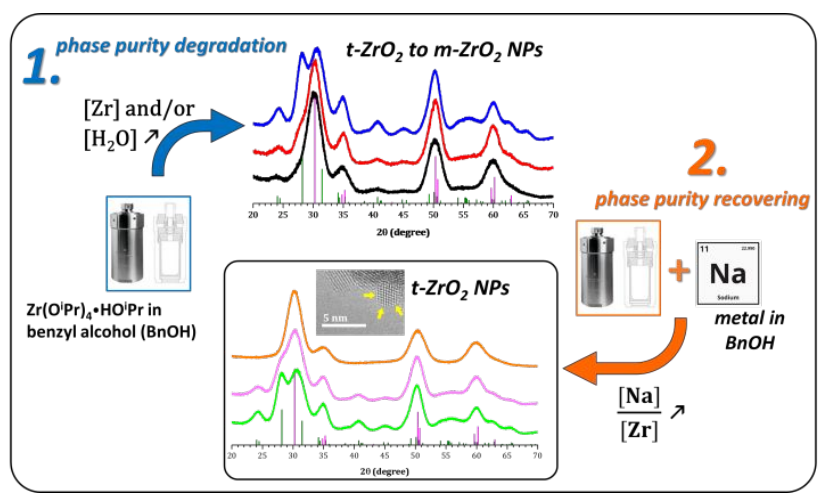

\section{Synopsis:}

Even under anhydrous conditions, the phase purity degradation of zirconia samples was observed jointly to the presence of water into the supernatant. Finally understood as the result of the alcohol dehydration supported by the surface of the nanoparticles and the release of insitu water, the tetragonal-to-monoclinic transformation was inhibited by the addition of sodium metal. Indeed, the disappearance of the monoclinic phase was observed provided that a sufficient sodium-to-zirconium ratio was ensured. 